\title{
Emine Sevgi Özdamar, Cécile Wajsbrot oder Berlistan, Parlin und die Bedrohungen des Lebens
}

Auf die Frage, ob man ihre Flucht vor der Militärdiktatur in der Türkei nach Deutschland zugleich auch als eine Flucht in die deutsche Sprache verstehen könne, antwortete Emine Sevgi Özdamar im November 2004 mit einem klaren Statement:

\begin{abstract}
Ja. Man erzählt immer, dass man in der Fremde die Muttersprache verliert. Ich denke, man kann auch die Sprache im eigenen Land verlieren. In schlimmen Zeiten macht die Sprache eine schreckliche Erfahrung. Ich hatte das Gefühl, dass die türkischen Wörter während der Militärputschzeit krank wurden. Ich hatte das Gefühl, dass ich in meiner Sprache sehr, sehr müde geworden war. ${ }^{1}$
\end{abstract}

Diese Passage stammt aus einem Interview, das aus Anlass der Verleihung des Kleist-Preises mit der am 10. August 1946 im türkischen Malatya geborenen, in Istanbul und Bursa aufgewachsenen Autorin Emine Sevgi Özdamar geführt wurde. Aber nicht nur dieses Statement, sondern auch ihr heute als Trilogie vorliegendes Romanwerk verdeutlicht die schmerzhaften Erfahrungen mit der eigenen Muttersprache, die für sie im Zeichen der türkischen Militärdiktatur stand und unter dem Druck eines ,alten“ Autoritarismus abgewirtschaftet hatte. So fragt die in ihrer türkischen Muttersprache unglücklich gewordene Ich-Erzählerin aus Seltsame Sterne starren zur Erde: „Wie lange braucht ein Wort, um wieder gesund zu werden? Man sagt, in fremden Ländern verliert man die Muttersprache. Kann man nicht auch in seinem eigenen Land die Muttersprache verlieren?"2 Diese Sätze machen zugleich darauf aufmerksam, dass Muttersprache und Vaterland im Schreiben dieser vielfach ausgezeichneten Schriftstellerin, die bereits im Alter von zwölf Jahren erstmals auf der Bühne stand und eine internationale Schauspielausbildung vorweisen kann, nicht voneinander getrennt werden können.

Die Sprache ihrer Literatur bildet ein System kommunizierender Röhren in einem zugleich translingualen und transkulturellen Sinne. Denn der Schock einer durch den Militärputsch von 1971 ausgelösten Erkrankung und Störung aller Lebens- und Kommunikationsbereiche beförderte bei Sevgi Özdamar eine heilsame und literarisch wirkungsvolle Querung verschiedener Sprachen und Kulturen. Auch wenn sie die Schauspielschule in Istanbul abbrechen musste und

1 Özdamar, Emine Sevgi: „Wir wohnen in einer weiten Hölle“ (Interview mit Nils Minkmar). In: Frankfurter Allgemeine Sonntagszeitung (Frankfurt am Main) 47 (21.11.2004), S. 23.

2 Özdamar, Emine Sevgi: Seltsame Sterne starren zur Erde. Wedding - Pankow 1976/77. Köln: Kiepenheuer \& Witsch 2003, S. 23. 


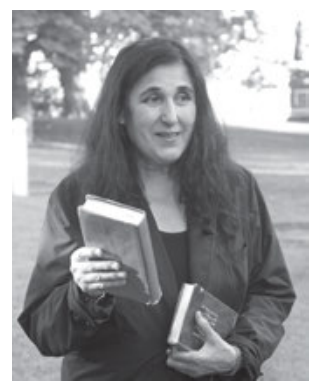

Abb. 132: Emine Sevgi Özdamar (Malatya, 1946).

nach Deutschland flüchtete, wo sie 1965 in West-Berlin zunächst in einer Elektrofabrik arbeitete, ermöglichte ihr diese internationale Dimension ihres schauspielerischen Tuns, das sie bald in Frankreich und Ost-Berlin komplettierte, doch einen Lebensweg, der im Zeichen hoher Kreativität stand und steht. Denn wie keine andere Schriftstellerin verkörperte Özdamar schon früh den Typus jener Literaturen ohne festen Wohnsitz, die zwar fast so alt sind wie die Literatur selbst, gerade aber während der vierten Phase beschleunigter Globalisierung und in unserer nach dieser Globalisierungsphase liegenden Zeit immer wichtiger werden. Es bleibt dabei: Innerhalb der Literaturen der Welt kommt den Literaturen ohne festen Wohnsitz eine stetig wachsende Bedeutung zu.

Die doppelten Türen im Titel von Özdamars 1992 erschienenem Romanerstling - Das Leben ist eine Karawanserei hat zwei Türen aus einer kam ich rein aus der anderen ging ich raus ${ }^{3}$ - machen unverkennbar auf eine zentrale Tatsache im Schaffen der unter anderem unter der Regie von Benno Besson, Claus Peymann oder Hark Bohm arbeitenden Akteurin aufmerksam. Räume und Bewegungen einschließlich aller Lebenszeiträume - werden dort stets als Transiträume, als Durchgangsorte gedacht, die über mehr als einen Ausgang, mehr als ein Bezugsund Kommunikationssystem verfügen. Nicht anders ist die mobile Architektur ihrer (literarischen) Landschaften und insbesondere Städte zu verstehen. Emine Sevgi Özdamar entschied sich 1986 für Berlin und ein Leben als freie Schriftstellerin, ohne ihre Theaterleidenschaft völlig aufzugeben. So aber traten neben das prägende Istanbul die beiden Seiten einer geteilten Stadt, die freilich ihre Teilung - aller Unkenrufe zum Trotz - größtenteils hinter sich gelassen hat.

3 Özdamar, Emine Sevgi: Das Leben ist eine Karawanserei hat zwei Türen aus einer kam ich rein aus der anderen ging ich raus. Köln: Kiepenheuer \& Witsch ${ }^{4} 1999$; vgl. zu den nachfolgenden Überlegungen auch ausführlich meinen Beitrag: Über die Brücke Unter den Linden. Emine Sevgi Özdamar, Yoko Tawada und die translinguale Fortschreibung deutschsprachiger Literatur. In: Arndt, Susan / Naguschewski, Dirk / Stockhammer, Robert (Hg.): Exophonie. Anders-Sprachigkeit (in) der Literatur. Berlin: Kulturverlag Kadmos 2007, S. 165-194. 
Als Begegnungsorte unterschiedlichster Kulturen spielen Städte und Großstädte im literarischen Oeuvre der einst ohne jede Kenntnis der deutschen Sprache aus der Türkei nach Deutschland gekommenen Schriftstellerin eine überragende Rolle. Vor einer genaueren Analyse von Özdamars Stadtentwürfen sollten wir nicht vergessen, dass der gesamte Text von Das Leben ist eine Karawanserei von ständigen Ortswechseln und Migrationen, aber auch von ständigen diatopischen und diastratischen Sprachwechseln in unterschiedlichen türkischen Städten geprägt ist. Zwischen dem Soldatenzug, mit dem gleich zu Beginn die mit der Ich-Erzählerin schwangere Mutter tief hinein nach Anatolien fährt, und dem „Hurenzug“, ${ }^{4}$ mit dem sich das mittlerweile etwa achtzehnjährige Mädchen auf den Weg nach WestBerlin macht, punktieren intralinguale ${ }^{5}$ Übersetzungsprozesse die Ortswechsel der Familie innerhalb der Türkei. Im Kontext dieser Migrationen werden gerade dialektale Besonderheiten der türkischen Sprache immer wieder diskutiert. So sind in die translokalen und transregionalen Migrationen innerhalb der Türkei bereits die transnationalen Bewegungen der Erzählerin auf eben jene Weise eingeschrieben, wie die intralingualen Übersetzungsprozesse stets schon auf die späteren translingualen Entwicklungen vorausweisen. Bewegungen zwischen Orten und Ländern, zwischen den Kulturen, Bewegungen zwischen den Sprachen prägen Leben, Denken und Schreiben dieser großen deutschsprachigen Schriftstellerin.

Versuchen wir, verschiedene Fäden unserer Argumentation in den bisherigen Sitzungen unserer Vorlesung zusammenzuführen: Dislokation und Heterotopie als ständiges Springen zwischen den Orten lassen eine Literatur ohne festen Wohnsitz entstehen, die - als querliegendes Konzept - weder in Kategorien wie „Nationalliteratur“ oder „Migrationsliteratur“ noch in solchen der „Weltliteratur“ aufgeht. Eine Fortschreibung der deutschsprachigen Literatur in diesem Sinne ständiger Dislokation wäre vielleicht am besten als „F:ortschreibung“ $\mathrm{zu}$ bezeichnen, ${ }^{6}$ insofern sie die Orte der Literatur im mehrfachen Sinne weiterschreibt, vervielfacht und verrückt. In jedem Falle macht Özdamars Entfaltung ihrer Literatur ohne festen Wohnsitz parallel zur vierten Phase beschleunigter Globalisierung auf das Ungenügen einer Philologie aufmerksam, welche ihren Gegenstand in Nationalliteratur und Weltliteratur aufteilt. Es besteht keinerlei Zweifel: Unser Modell einer viellogischen Auffassung verschiedener Literaturen der Welt ermög-

4 Özdamar, Emine Sevgi: Das Leben ist eine Karawanserei, S. 379.

5 Zur Kulturtechnik intralingualer Übersetzung vgl. Jakobson, Roman: On linguistic aspects of translation. In (ders.): Selected Writings. II. Word and Language. Den Haag - Paris: Mouton 1971, S. 260.

6 Ich greife mit dieser Schreibweise ein poetisches Verfahren auf, das ich der Lyrik José F. A. Olivers entnehme. Zur Funktion des Doppelpunktes bei Oliver vgl. Ette, Ottmar: ÜberLebenswissen. Die Aufgabe der Philologie. Berlin: Kulturverlag Kadmos 2004, S. 247. 
licht uns ein Analysieren, Deuten und Interpretieren literarischer Texte weltweit, welches sich aus den Zwängen alter, überkommener und irreführender Kategorien befreit hat.

Betrachten wir die unterschiedlichen Choreographien auf Ebene der Handlungsstrukturen, so sind Pendelbewegungen ein charakteristischer Bestandteil der Bewegungsmuster in den Romanen Emine Sevgi Özdamars. So heißt es etwa im 1998 unter dem Titel Die Brücke vom Goldenen Horn erschienenen zweiten Teil ihrer Romantrilogie mit Blick auf Istanbul, jene zwei Kontinente miteinander verbindende Stadt am Bosporus: „Zwischen Asien und Europa gab es damals, 1967, noch keine Brücke. Das Meer trennte die beiden Seiten, und wenn ich das Wasser zwischen meinen Eltern und mir hatte, fühlte ich mich frei. " ${ }^{7}$ Und wenig später heißt es: „Die asiatische und die europäische Seite in Istanbul waren zwei verschiedene Länder." ${ }^{8}$ Zwischen diesen beiden Ländern, zwischen diesen beiden Seiten der Stadt aber siedeln sich alltägliche Pendelbewegungen der Protagonistin an.

Das ständige Oszillieren zwischen diesen beiden ,Welten“ wird zur alltäglichen, rasch selbstverständlich gewordenen Übung, die es der Ich-Erzählerin erlaubt, ihren professionellen, intellektuellen und sexuellen Bildungsroman oder - mit Blick auf Die Brücke vom Goldenen Horn - ,Entwicklungsroman“9 wo nötig fernab jeder Kontrollmöglichkeit durch die Eltern fortzuschreiben. Es ist eine Dialektik von Nähe und Ferne, welche die Entwicklung der Ich-Figur sicherstellt und gleichsam in der Grundstruktur der Stadt verankert.

Die Trennung zwischen Asien und Europa erscheint auf den ersten Blick fundamental; doch die Überwindung dieser geographischen und vor allem geopolitischen Aufspaltung gehört zugleich zu einer Alltagserfahrung, welche die Protagonistin mit Tausenden von Pendlern auf den Schiffen teilt. Wie Weberschiffchen halten die Dampfer die beiden Seiten Istanbuls im Leben der jungen, gerade erst zwanzigjährigen Frau zusammen und bilden das Gewebe, den Text eines ständigen Über-Setzens und Übersetzens, das sich nicht im „Dazwischen“ fest etabliert, sondern den Zwischenraum zu einem Bewegungsraum macht, der vom unablässigen raumzeitlichen Hin und Her erfüllt wird. In den Strukturen und Strukturierungen der Stadt ist ein Schreiben angelegt, das sich in dieser Welt zwischen beiden Welten situieren wird.

7 Özdamar, Emine Sevgi: Die Brücke vom Goldenen Horn. Köln: Kiepenheuer \& Witsch 1998, S. 222.

8 Ebda.

9 Vgl. hierzu Ackermann, Irmgard: Emine Sevgi Özdamar. In: Kritisches Lexikon zur deutschsprachigen Gegenwartsliteratur. 62. Nachlieferung. München: Edition text-kritik 1999, S. 5. 
Doch damit nicht genug! Es ist verblüffend, wie präzise und hintergründig zugleich dieses in Die Brücke vom Goldenen Horn vor Augen geführte Modell der Stadt Istanbul wenige Jahre später auf das Berlin Mitte der siebziger Jahre mit seiner scharfen Trennung durch die Berliner Mauer in ein Ost- und ein West-Berlin übertragen wird. Die geteilte, noch immer in unterschiedliche Sektoren zerfallende Stadt ist im dritten, 2003 veröffentlichten Roman Seltsame Sterne starren zur Erde gleichfalls in zwei Teile gespalten, die sich wie Asien und Europa als Ostblock und Westblock, als zwei unterschiedliche, auf den ersten Blick unversöhnlich durch feindliche Systeme und Schussanlagen voneinander getrennte Städte und ,Länder gegenüberstehen. An die Stelle der Meerenge des Bosporus ist die Berliner Mauer getreten, an jene der Schiffe die Berliner S-Bahn, welche die junge Türkin mit Touristenvisum und ohne festen Wohnsitz in ebenfalls zwanzigminütiger Fahrt von der einen, mit unzähligen Graffiti beschrifteten Seite der Mauer auf die andere bringt. Denn die Mauer ist für die jugendliche Migrantin mit türkischem Pass kein unüberwindliches Hindernis: Ihre Überwindung und Querung ist eine fast alltägliche Erfahrung, die bald zur Routine, also zum „kleinen Weg“, wird.

Denn die Lebensbedingungen des Alter Ego von Emine Sevgi Özdamar sind spektakulär und alltäglich zugleich. Bis zur Erteilung eines dreimonatigen Visums durch die Behörden der Hauptstadt der DDR lebt die aus der Untersuchungshaft in der Türkei freigekommene Schauspielerin, die ihren Beruf in ihrem Heimatland nicht mehr ausüben darf, in einer West-Berliner Wohngemeinschaft, die ihr - wie bei den Eltern in Istanbul - kostenlos Unterkunft und ,Familienanschluss“ bietet. Trotz aller wichtigen Erfahrungen, die ihr in der liebe- und humorvoll (und nicht ohne eine Prise Spott) gezeichneten Wohngemeinschaft im Wedding zuteilwerden, findet sie ihre eigentliche Erfüllung auf der Ost-Berliner Seite. Dort kann sie in ,ihre' Welt des stark von Brecht geprägten Theaters eintauchen und findet nach Erteilung ihres Visums Unterkunft bei einer jungen Frau in einem nicht weniger detailgetreu nachempfundenen Setting. Die Schauspielerei steht im Mittelpunkt ihres Lebens; und der Schauspielerin erscheinen alle Elemente auf der Bühne dieses Lebens wie inszeniert, wie für sie in Szene gesetzt.

Wieder sind auf den ersten Blick die beiden unterschiedlichen ,Ländern“ und ,Blöcken‘ zugehörigen Teile der Stadt klar voneinander geschieden und durch einen Todesstreifen voneinander getrennt. Doch bleiben sie wie in Istanbul nicht nur durch Telefonanrufe und gelegentlichen Blickkontakt - zwischen östlicher und westlicher wie zuvor zwischen asiatischer und europäischer Seite - miteinander in Verbindung, sondern werden durch tagtägliche S-Bahnfahrten miteinander eng verwoben. Bald kennt unsere Protagonistin die Berliner Mauer von der westlichen wie von der östlichen Seite, die Grenzabfertigungen sind für sie Routine. Ihr Berlin ist für sie keine geteilte, sondern eine eng verwobene Metropole, in der das Theatralische im Mittelpunkt steht. 
Diese Ich-Erzählerin, welche sich nach zwischenzeitlicher Heirat in Istanbul von ihrem Mann getrennt hat, pendelt zwischen verschiedenen Wohnungen und gelegentlich auftauchenden und wieder verschwindenden Liebhabern, bleibt aber ohne festen Wohnsitz und - ganz wie im Leitmotiv des titelgebenden Gedichts von Else Lasker-Schüler - „Mit brennenden Armen die Liebe suchend“. ${ }^{10}$ In diesem Vers bündeln sich ein weiteres Mal die Sehnsucht nach Liebe und die Suche nach dem eigenen Ich. Dass der direkt in den Titel gespiegelte Intertext einer jüdisch-deutschen Autorin entstammt, die vor den Nationalsozialisten ins Ausland fliehen musste und gleichsam ohne ihr Zutun an jener jüdischen Literatur weiterschrieb, die eine Literatur ohne festen Wohnsitz bildet, erscheint keineswegs als Zufall. Ein Schreiben zwischen den Welten ist all diesen Texten und Traditionen eingeschrieben.

Berlin ist in vielfachem Sinne die Stadt ihrer Sehnsucht - und ist doch immer noch auch Istanbul. Istanbul ist in Berlin präsent, so wie Berlin in Istanbul gegenwärtig wird. Es bildet sich etwas heraus, das wir „Berlistan“ nennen können, eine doppelt geteilte Stadt, die auf doppelte Weise eng miteinander verwoben wird. So entsteht eine verdoppelte Stadtlandschaft, die genügend Raum für ein ZwischenWeltenSchreiben und ein ZwischenWeltenLeben lässt.

Innerhalb des Berliner Stadtraums erscheint im Roman ebenso ein nahezu kontaktloses multikulturelles Nebeneinander wie ein breites Spektrum interkultureller Beziehungen, also wechselseitiger Kontakte zwischen verschiedenen sprachlichen, kulturellen oder auch soziopolitischen Gruppen und Individuen. Auch im weiteren Fortgang ihres Lebensweges spielen Übersetzungen im privaten wie beruflichen, im mündlichen wie schriftlichen Bereich der Protagonistin eine wichtige Rolle, übersetzt sie doch für die Firma Siemens ${ }^{11}$ zwischen Deutschen und Türken sowie für ihre in der Türkei einsitzenden Schauspielerfreunde die Notizen des Brecht-Schülers Benno Besson. Die vormalige ,Botin` zwischen zwei Gruppen, zwischen zwei voneinander getrennten sprachlichen (und zugleich sozialen, kulturellen und politischen) Gemeinschaften findet zunehmend zu ihrer eigenen, von ständigen Kreuzungen geprägten Sprache. Diese Literatursprache ist ebenso transareal wie transkulturell geprägt und vermag sich nicht mehr nur an einer Sprache, einer Kultur, einer Stadt zu orientieren. In der einen Sprache ist die andere Sprache stets präsent ...

Untersucht man die Raum- und Bewegungsstrukturen genauer, die in den Schriften Emine Sevgi Özdamars entfaltet werden, so zeigt sich, dass wie auf Ebene der Worte, unter denen andere Worte sichtbar und hörbar werden, auch

10 Özdamar, Emine Sevgi: Seltsame Sterne starren zur Erde, S. 9.

11 Vgl. hierzu ebda., u. a. S. $112 \mathrm{f}$. 
auf jener der Orte andere Orte auszumachen sind. So erscheint Istanbul ständig in Berlin - und zwar gerade nicht als „Klein-Istanbul“: Unter den Bewegungen in der Stadt werden vielmehr die früheren Bewegungen in einer anderen Stadt mit ihrer eigenen Choreographie, ihrer eigenen Logik sichtbar. Berlistan beginnt $\mathrm{zu}$ leben. Entscheidend dabei ist: Alle Prozesse und Entwicklungen des Verstehens werden in Özdamars Schreiben nicht nur verräumlicht, sondern zugleich vektorisiert. Denn in jeder aktuellen Bewegung sind die vorausgegangenen Bewegungen immer schon akkumuliert beziehungsweise gespeichert und weisen auf künftige Bewegungen voraus: Nichts ist statisch.

In diesem Sinne erfüllt sich zumindest zum Teil die poetische Prophezeiung ihres Mannes, den die Ich-Erzählerin in seinem Waldhaus auf der asiatischen Seite in Istanbul zurückließ. Er hatte seiner die Stadt am Bosporus scheinbar hinter sich lassenden Frau diese Worte mitgegeben:

\footnotetext{
„Du wirst keine neuen Länder entdecken, keine anderen Meere.

Die Stadt wird dir folgen. Du wirst durch dieselben Straßen

Streifen, in denselben Vierteln alt werden. ${ }^{\text {"12 }}$
}

Folglich verwundert es nicht, dass auch das Waldhaus, das man von der europäischen Seite aus sehen konnte, ${ }^{13}$ in Berlin sein Gegenstück findet: So wird die Protagonistin - kurz nachdem sie von ihrem Istanbuler Waldhaus träumte (in dem sie mit ihrem Mann so glückliche Monate verbrachte) - vom Ost-Berliner Kollegen eines Kamerateams und dessen Frau in deren „einsames Waldhaus“14 eingeladen. Berlin und Istanbul bilden dieselbe Stadt: Das Fremde erscheint im Eigenen, das Eigene im Fremden, beide sind ein und dasselbe Berlistan und bilden ein gemeinsames mentales Mapping, welches die urbanen Landschaften nicht länger getrennt voneinander erfasst.

Dies bedeutet nicht, dass die beiden Städte identisch wären, also aufgehört hätten, voneinander getrennt zu sein. Berlistan bildet eine Stadt, die zwei Städte ist; beide Städte bilden jedoch zugleich für die Protagonistin dieselbe Stadt. Denn in Berlin sucht sie sich krampfhaft damit zu beruhigen, dass sie nun ,in einem anderen Wald“, ${ }^{15}$ in einem anderen Waldhaus sei. Filmtechnisch gesprochen haben wir es hier mit einer der unzähligen Überblendungen zu tun, die Istanbul in Berlin auf allen Ebenen und mit allen Sinnen zum Vorschein bringen. Nicht

12 Özdamar, Emine Sevgi: Seltsame Sterne starren zur Erde, S. 56 (Es handelt sich hier um einen von der Autorin vermerkten Auszug aus einem Gedicht von Konstantinos Kavafis).

13 Ebda., S. 28.

14 Ebda., S. 113.

15 Ebda. 
nur, dass selbst ein Wasserrohrbruch Unter den Linden die Erzählerin an einen Lindenbaum neben ihrem Istanbuler Waldhaus erinnert, wobei diese Erinnerung in einer für die Ich-Figur charakteristischen Assoziationskette zugleich mit Literatur - einem türkischen Dichterfreund - und Liebe verknüpft wird. ${ }^{16}$ Alles beginnt ganz alltäglich und fast nebensächlich, legt aber die tiefen Beziehungen innerhalb Berlistans frei:

Heute Abend soll Unter den Linden ein Rohr geplatzt sein. Die Straße war überschwemmt. Überall standen Feuerwehrautos und Polizeiautos unter den Linden. Auch am Waldhaus in Istanbul gab es einen Lindenbaum. Darunter saß oft ein Dichterfreund, seine Haare waren voller Blüten. In der Türkei sagt man, dass der Geruch der Lindenblüten junge Mädchen verrückt macht. ${ }^{17}$

Zwar fällt es der Protagonistin immer wieder auch einmal schwer, die beiden Teile Berlins zusammenzudenken, doch erscheint ihr die Grenze zwischen West- und Ost-Berlin schon bald wie in Istanbul als „ein großes Meer“"18 - und dieses lädt zum Über-Setzen, zum queren und kreuzen ein. Nicht nur die „Morgenstimmung“, sondern auch der „Geruch“ auf den Straßen von Ost-Berlin erinnert sie unmittelbar an die türkische Metropole: ${ }^{19}$ „ein Geruch von Kohle und Autoabgasen wie in Istanbul“, ${ }^{20}$ so dass sich ein gemeinsamer transarealer „Smellscape“ herausbildet, eine alles verbindende typische Geruchslandschaft. Und selbst das Sehen eines US-amerikanischen Films mit Rod Taylor erinnert sie daran, dass dies einer ihrer „Kindheitsfilme aus Istanbul“21 war: Immer wieder stellen sich die Bilder, Geräusche und Gerüche aus der Stadt am Bosporus ein, verwandeln Berlin in Istanbul und projizieren Berlin an die Grenzen Europas.

Durch jeden Gegenstand, durch jeden Sinneseindruck kann der Überblendungsmechanismus zwischen Istanbul und Berlin ausgelöst und somit - nicht nur mit Blick auf die Überlagerungen zwischen Die Brücke vom Goldenen Horn und Seltsame Sterne starren zur Erde - eine „Brücke Unter den Linden“ geschaffen werden. In der einen Stadt ist die andere stets gegenwärtig: Sie ist mit ihrer Verbindung von Liebe und Literatur der Migrantin gefolgt, ist nicht vertikal beziehungsweise archäologisch mit älteren Schichten Berlins, sondern horizontal mit anderen Räumen und Richtungen verbunden. Noch einmal: Beide Städte sind

16 Ebda., S. $95 \mathrm{f}$.

17 Ebda.

18 Ebda., S. 18.

19 Ebda., S. 81.

20 Ebda.

21 Ebda., S. 123. 
nicht miteinander identisch, verschmelzen nicht miteinander, doch zugleich sind sie untrennbar in einem einzigen Berlistan gegenwärtig.

Dieses In-Ein-Ander-Schreiben beider Städte und ihrer jeweiligen Zweiteilung, die durch die Reisebewegungen der Protagonistin immer wieder neu miteinander verflochten werden, lässt sich auch in der Überlagerung von Wohnstrukturen beobachten. In Berlins türkischem Frauenwohnheim, das während des ersten Berlinaufenthalts ganz bewusst als „Frauenwonaym“22 fremdgeschrieben wurde, finden sich bereits die Strukturen des Wohnheims, das die Ich-Erzählerin während ihres zweiten Aufenthalts in der Stadt an der Spree kennenlernen wird. Die Lebensverhältnisse in diesen Wohnheimen mit ihren klaren Unterteilungen und sexuellen wie geschlechterspezifischen Grenzlinien finden ihr parodistisches und zugleich paradoxes Gegenstück in der Weddinger Wohngemeinschaft, wo die Neuangekommene gleich zu Beginn nicht nur - wie schon im Frauenwohnheim - das Geschirr spülen darf, sondern nachdrücklich auf das Verbot jeglicher Pärchen-Beziehungen aufmerksam gemacht wird. Die kollektiven Wohnformen, die im Verlauf der Romane immer wieder auftauchen, bilden eine eigene Welt, gleichsam einen urbanen Transitraum, der für einen begrenzten Zeitraum zu einem „Phalanstère“ werden kann. Nicht allein die Lebensformen, sondern auch die Lebensnormen, an die sie sich zu halten hat, werden ihr drastisch vor Augen geführt.

So bemerkt die Erzählerin erst beim Versuch einer Freundin, das Frauenwohnheim zu verlassen, dass es Berlin „für uns bis jetzt nicht gegeben hatte“: ${ }^{23}$ „Wir hatten unser Wonaym, und dieses Wonaym war nicht Berlin. Berlin begann erst, wenn man aus dem Wonaym herausging.“24 Die jeweiligen Lebensnormen unterscheiden sich in radikaler Weise voneinander: Und diese Unterschiede zeigen sich nicht nur innerhalb Istanbuls oder zwischen Istanbul und Berlin, sondern auch in Berlin selbst. Also doch eine Stadt der Parallelgesellschaften?

Worte unter Worten, Orte unter Orten, Bewegungen unter Bewegungen, Wohnformen unter Wohnformen, Ereignisse unter Ereignissen, Städte unter Städten. Die Romane Das Leben ist eine Karawanserei, Die Brücke vom Goldenen Horn und Seltsame Sterne starren zur Erde erzählen zwar weitgehend chronologisch eine Lebensgeschichte und lassen auch die dazu gehörigen geschichtlichen Kontexte von der widersprüchlichen Modernisierung der Türkei durch Atatürk über die Erfahrungen der 68er-Generation bis hin zu den Spätformen des Kalten

22 Diese Schreibweise findet sich bereits im Titel des ersten Kapitels des ersten Teils von Özdamar, Emine Sevgi: Die Brücke vom Goldenen Horn, S. 11.

23 Ebda., S. 63.

24 Ebda. 
Krieges und - zumindest perspektivisch - bis zum „Deutschen Herbst“ und zum Fall der Berliner Mauer aufscheinen. Doch zeigt es sich, dass es neben diesen syntagmatischen Elementen vor allem sich ständig überlagernde paradigmatische Strukturierungen sind, welche entscheidend die Verstehens-Prozesse im Roman bestimmen. Die Überblendungstechnik des Ineinander-Schreibens führt vor Augen, dass die europäische, auf beiden Seiten der Brücke vom Goldenen Horn liegende Seite Istanbuls nicht ohne die asiatische, West-Berlin nicht ohne OstBerlin, Berlin nicht ohne Istanbul und Deutschland nicht ohne die Türkei gedacht werden können. Berlistan ist eine mentale Projektion, die längst zur Realität geworden ist, die sich wie alle Realität aus friktionalen Quellen speist.

Dabei geht es nicht um interkulturelle Vermittlung, sondern um transkulturelle Verschränkung und Durchquerung, eine verschiedene Kulturen querende Choreographie von Bewegungen, die keineswegs euphorisch nur als lustvoll, sondern nicht selten als höchst spannungsvoll und schmerzhaft porträtiert werden. Nein, Berlistan ist keineswegs nur lustvolle Vereinigung! Aber wie alle liebevolle Lust vermittelt sie allen Beteiligten tiefe Erkenntnis.

Zweifellos lassen sich hierbei fraktale, vielfach gebrochene und zugleich insuläre Muster und Strukturen feststellen, wie sie in den, aber auch für die Literaturen ohne festen Wohnsitz von grundlegender Bedeutung sind. Dies bedeutet zugleich, dass sich die bereits mehrfach benannte Vektorisierung aller Bezüge nicht in einem kontinuierlichen, sondern in einem diskontinuierlichen, vielfach gebrochenen post-euklidischen Raum vollzieht, ${ }^{25}$ in den sich die Strukturen und Strukturierungen der Stadt - oder besser: der Städte - eintragen. Es handelt sich um einen diskontinuierlichen archipelischen Bewegungs-Raum, der nicht den statischen Zusammenhalt, sondern die mobile Vielverbundenheit vor Augen führt. Denn Berlistan ist eben letzteres: offene, relationale Vielverbundenheit!

Mit Bedacht stellte die Autorin nicht zwei nationale, sondern vor allem zwei urbane Räume in den Mittelpunkt ihres Romanzyklus, die an den Nahtstellen west-östlicher Geschichte liegen und diese Lage zwischen zwei Kontinenten und zwei Machtblöcken bis in ihre Topographie und Infrastruktur hinein reflektieren. Das Raummodell, das in diesem Romanzyklus entfaltet wird, hat nichts mit der Kontinuität statischer nationaler Räume zu tun, sondern eröffnet Bewegungsräume, die nur bewegungsgeschichtlich und damit vektoriell $\mathrm{zu}$ erfassen sind. Bilden diese urbanen Räume nicht Inseln, ja mehr noch Archipele aus, die in ihrer Relationalität neue vektorisierte Verstehensbewegungen ebendieser Räume

25 Vgl. hierzu Ette, Ottmar: Von Inseln, Grenzen und Vektoren. In: Braig, Marianne / Ette, Ottmar / Ingenschay, Dieter / Maihold, Günther (Hg.): Grenzen der Macht, Macht der Grenzen. Frankfurt am Main: Vervuert 2005, S. 135-180. 
vorführen? Wir wollen derartige Vorstellungen am Beispiel eines anderen Romanzyklus vor Augen führen, der sich in seiner Entfaltung ebenfalls über die Grenzen zwischen postmodernem und nachpostmodernem Schreiben hinwegbewegt und neue Horizonte literarischer Entwicklung aufzeigt.

Mit ihrem im August 2008 erschienenen Roman L'Ile aux musées hat die französische Schriftstellerin Cécile Wajsbrot, ${ }^{26}$ eine seit vielen Jahren zwischen der französischen und der deutschen Hauptstadt pendelnde Autorin, einen faszinierenden Erzähltext vorgelegt, welcher sich innerhalb eines Romanzyklus verortet, für den wir zum Abschluss unserer Vorlesung noch ein zweites Beispiel kennenlernen werden. Nicht von ungefähr steht im Herzen dieses Romans, der zu den schönsten der französischen Schriftstellerin zählt, eine Insel, die im Grunde viele Inseln ist.

Die 1954 in Paris geborene Autorin stammt aus einer jüdischen Familie, die sich aus Polen nach Frankreich geflüchtet hatte und später, nach dem Einmarsch deutscher Truppen, von den Nationalsozialisten in Frankreich verfolgt wurde. Diese tragische Familiengeschichte - der Großvater der Schriftstellerin wurde in Auschwitz ermordet - bildet eines der verschiedenen autobiographischen Grundmuster, in deren Webrahmen die Vielzahl beeindruckender Romane entstand, die aus der Feder der Literatin und Übersetzerin stammen, die an der Pariser Sorbonne Komparatistik studierte. Cécile Wajsbrots gesamtes literarisches Oeuvre gehört heute zu den herausragenden und maßgeblichen Schöpfungen einer französischen Literatur, die nach Jean-Paul Sartre und Simone de Beauvoir, nach dem Nouveau Roman, nach den großen Vertreter^innen der französischen Literatur- und Kulturtheorie mit dem Eintritt in die vierte Phase beschleunigter Globalisierung - wie besprochen - ihre herausragende Stellung verlor. Nicht ohne Verkrampfung und Verzweiflung suchte diese Literatur innerhalb der Welt französischsprachiger Literaturen nach neuen Wegen und Ausdrucksmöglichkeiten.

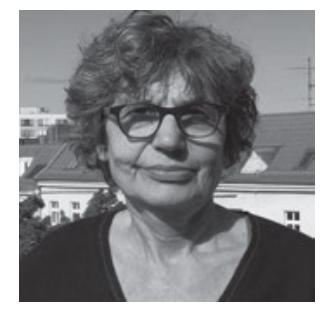

Abb. 133: Cécile Wajsbrot (Paris, 1954).

26 Vgl. den Sammelband von Böhm, Roswitha / Zimmermann, Margarete (Hg.): Du silence à la voix. Studien zum Werk von Cécile Wajsbrot. Göttingen: F\&R unipress 2010. 
Seit Mitte des ersten Jahrzehnts des neuen Jahrtausends, seit ihrem 2007 erschienenen Roman Conversations avec le maître, arbeitete Cécile Wajsbrot an einem Zyklus, der erst zwölf Jahre später - im Jahr 2019 - mit dem Band Destruction vollendet werden sollte. Der weit gespannte Zyklus Haute Mer umfasst neben dem Auftaktroman Conversations avec le maître (2007) ${ }^{27}$ die Romane L'Ile aux musées $(2008)^{28}$ Sentinelles (2013), ${ }^{29}$ Totale éclipse $(2014)^{30}$ sowie ihren 2019 erschienenen Abschlussroman Destruction ${ }^{31}$ und erstreckt sich über eine Schaffensperiode von deutlich mehr als einem Jahrzehnt. In jedem der einzelnen Romane dieses Zyklus einer Autorin, die zum Mitglied der Deutschen Akademie für Sprache und Dichtung sowie der Berliner Akademie der Künste gewählt wurde, steht eine andere künstlerische Ausdrucksform im Vordergrund. Ein literarisches Gestaltungsprinzip, das nicht allein große schöpferische Kraft besitzt, sondern es auch erlaubt, die spezifische Stellung der Literatur im Gefüge künstlerischer Medien gleichsam „en passant“ vor Augen zu führen und sinnlich nachvollziehbar zu machen. In L'Ile aux musées steht die Bildhauerkunst im Vordergrund, wobei uns das Leben der unterschiedlichsten Statuen nicht nur von außen, sondern gleichsam von innen gezeigt wird. Und dies erfolgt nicht in irgendeinem beliebigen räumlichen Setting, sondern an jenem Ort im Zentrum Berlins, an dem keine Menschen wohnen: der Museumsinsel.

Dabei ist die Berliner Museumsinsel nicht allein, sondern wird von der Pariser Stadtinsel ergänzt. Inmitten der die Romandiegese prägenden und so unterschiedlichen, ja gegensätzlichen (und eben dadurch komplementären, sich wechselseitig ergänzenden) Stadtlandschaften von Berlin und Paris, in deren Herzen sich mit der Ile de la Cité und der Museumsinsel jeweils eine Insel befindet, bilden die Blicke der Berliner Statuen Geflechte, die den achtlos unter oder neben ihnen vorbeieilenden Menschen verborgen und unbekannt bleiben. Denn wann schon blicken wir all jenen Statuen bewusst ins Gesicht, denen wir Tag für Tag unter die Augen kommen?

27 Wajsbrot, Cécile: Conversations avec le maître. Paris: Editions Denoël 2007.

28 Wajsbrot, Cécile: L'île aux musées. Paris: Editions Denoël 2008. Vgl. hierzu Ette, Ottmar: Cécile Wajsbrot: „L'Ile aux musées“ oder die verborgenen Choreographien der Anwesenheit. In: Böhm, Roswitha / Bung, Stephanie / Grewe, Andrea (Hg.): Observatoire de l'extrême contemporain. Studien zur französischsprachigen Gegenwartsliteratur. Tübingen: Gunter Narr Verlag 2009, S. 257-270.

29 Wajsbrot, Cécile: Sentinelles. Paris: Christian Bourgois 2013.

30 Wajsbrot, Cécile: Totale éclipse. Paris: Christian Bourgois 2014.

31 Wajsbrot, Cécile: Destruction. Roman. Paris: Le Bruit du Temps 2019. 
Eben hier tritt der Roman, der zweite des Zyklus, in sein Recht. Hat nicht die Literatur die Aufgabe, das durch seine Allgegenwart unsichtbar Gewordene wieder sichtbar zu machen? So heißt es schon im ersten Kapitel des Romans:

\begin{abstract}
Wir halten Wache, selbst wenn niemand auf uns achtet - vielleicht ist es sogar einfacher aufzupassen, wenn einem niemand zuschaut. Unser Schicksal ist seltsam. Bevor wir auf die Welt kommen, sind wir der Gegenstand von Verhandlungen, von Entscheidungen, von Umdeutungen, von Kompromissen, Sie schlagen sich um das Recht, uns Existenz zu geben, aber wenn wir erst einmal da sind, setzt sich unsere Präsenz durch und die Ihre verschwindet, die Rollen kehren sich um - Sie geraten dann zu Vorwänden und der Raum gehört uns.

Der Raum gehört uns und wir halten Wache. Wir sind aus Stein, aus Bronze, wir sind aus Granit oder Marmor, wir stehen auf Brücken, hoch oben auf Gebäuden oder vor Museen, wir stehen in den Gärten, als Vorzeichen, befinden uns in Nischen - freilich unbeweglich, mit festem Blick. ${ }^{32}$
\end{abstract}

Die Allgegenwart der so verschiedenartigen Statuen wird hier ebenso deutlich wie ihr immer wieder hervorgehobener Zeichencharakter. Ihre Beherrschung des Raumes bleibt den Menschen, die sie doch geschaffen haben, ebenso weitgehend verborgen wie ihre Beherrschung eines Zeit-Raums, der die Grenzen individuellen menschlichen Erlebens sprengt: Für lange Jahrhunderte sind wir da - „Nous sommes là pour des siècles“. ${ }^{33}$ Wie in vielen Romanen Cécile Wajsbrots - und es wäre ein Leichtes, die vielen intratextuellen Verweise und Anspielungen in L'Ile aux musées aufzuzeigen - wird in der Präsenz des Anderen, in den Tieren wie den Künsten, in der Schneeeule von Mémorial ${ }^{34}$ wie der Malerei der Romantik von Caspar-Friedrich-Strasse, ${ }^{35}$ eine Ander-Logik (der Kunst wie der Natur) greifbar und begreifbar. Sie übersteigt das Menschliche und die Koordinaten menschlichen Lebens bei weitem. Jenseits der Blicke der Menschen bilden die Blickgewebe der Statuen Beziehungen, die Räume und Zeiten queren und durchqueren.

Die Statuen haben ein langes Gedächtnis, ihr Erleben ist transhistorisch. Sie wissen von Schlössern und Palästen der Menschen, wissen auch von jenen repräsentativen Prachtbauten, die erbaut und später zerstört wurden, erzählen von der

32 Wajsbrot, Cécile: L’Ile aux musées. Roman. Paris: Editions Denoël 2008, S. 10; vgl. eine ausführlichere Analyse dieses Romans in Ette, Ottmar: Cécile Wajsbrot: „L'lle aux musées“ oder die verborgenen Choreographien der Anwesenheit. In: Böhm, Roswitha / Bung, Stephanie / Grewe, Andrea (Hg.): Observatoire de l'extrême contemporain. Studien zur französischsprachigen Gegenwartsliteratur. Tübingen: Gunter Narr Verlag 2009, S. 257-270.

33 Wajsbrot, Cécile: L'Ile aux musées, S. 11.

34 Vgl. Wajsbrot, Cécile: Mémorial. Paris: Zulma 2005.

35 Vgl. Wajsbrot, Cécile: Caspar-Friedrich-Strasse. Paris: Zulma 2002. 
Geschichte der Kunstsammlungen im Louvre und dem Alten Museum, ${ }^{36}$ kennen die Geschichte und viele Geschichten, ohne selbst auch nur im Geringsten ins Historische abgeschoben und damit historisch geworden zu sein. Sie sind weder auf die bloße Gegenwart ihrer Gegenständlichkeit noch auf die Gegenständlichkeit ihrer Gegenwart reduzierbar: „Ihr seid im Präsens. Wir sind in der Präsenz. - Vous êtes dans le présent. Nous sommes dans la présence. “37

Angesichts dieser Präsenz jenseits des Präsentischen erscheint die Gegenwart, das Präsens der Menschen als prekär und flüchtig. Vor dem Hintergrund einer Geschichte von Kriegen und Katastrophen wird eine Zukunft des Planeten vorstellbar, aus der die Menschen verschwunden sind, so wie eine Vergangenheit erscheint, in der die Menschen noch nicht gegenwärtig waren: „Avant, il y avait une île, avant encore, des marais, la terre cernée par les eaux.“38 Ja, es gab eine Geschichte unseres Planeten vor dem Menschen! Und es könnte sein, dass es wieder eine Geschichte geben wird, welche ohne den Menschen, ohne Präsens und Präsenz der Menschheit auskommen wird. Für die Statuen jedenfalls ist der Mensch verzichtbar, ,wegdenkbar' geworden. Auch in diesem literarischen Text des 21. Jahrhunderts kommt eine Konvivenz auf dem Planeten Erde ohne den Menschen aus - sei es auf Grund einer ökologischen oder einer atomaren Katastrophe.

Wie entstand der Kosmos, wie entstand ein Universum von Ordnung und Schönheit? Am Anfang der Genesis, dieser Genesis, war das Wasser und war das Land. Und der Tag zeichnet sich schon ab, an dem die Schiffe der Menschen dieses Wasser nicht mehr befahren, die Flugzeuge der Menschen den Himmel über dieser Erde nicht mehr durchqueren werden. ${ }^{39}$ Allein die Statuen werden bleiben als „die letzten Zeugen Ihrer Sehnsüchte“. ${ }^{40}$ Sie verwandeln sich in die eigentlichen Bewohner der Stadt, die eigentlichen Subjekte im urbanen Zeichenreich der Menschen, das von einem Leben jenseits des Menschen jederzeit wieder in Besitz genommen werden kann. Wohl aus diesem Grund sind alle menschlichen Figuren im Roman nur schemenhaft und flüchtig gezeichnet, Namenlose auf der Suche nach ein wenig Liebe und Zusammenleben. So filigran sie skizziert sind, so schemenhaft können sie auch wieder verschwinden, wie Zeichen im Sand, wie ein Gesicht am Strand, um Michel Foucaults berühmtes und in unserer Vorlesung zitiertes Ende von Les mots et les choses anzuführen. Von Dauer ist ihre Welt nicht:

36 Vgl. Wajsbrot, Cécile: L'Ile aux musées, u. a. S. 12.

37 Ebda., S. 11.

38 Ebda.

39 Ebda., S. 229.

40 Ebda.: „les derniers témoins de vos aspirations.“ 
Ihre flüchtigen Konturen sind in den Augen der Statuen nur Spuren, nur Schatten einer Choreographie der Anwesenheit im nur Präsentischen.

Cécile Wajsbrots Roman L'Ile aux musées gibt seinem Lesepublikum Elemente einer Geschichte der Berliner Museumsinsel an die Hand, die gleichsam transhistorisch perspektiviert werden. Im historiographischen Diskurs klingt diese Entstehungsgeschichte - ungeachtet aller west-ost-ideologischen Orientierungen etwa so:

Die im Zentrum Berlins gelegene Insel, östlich begrenzt von der Spree und westlich von einem ihrer Nebenarme, dem Kupfergraben, war zu Beginn des 15. Jahrhunderts jener geographisch günstig gelegene Ort zwischen den beiden märkischen Handelsstädten Berlin und Cölln, auf dem sich die 1417 in den Besitz der Mark Brandenburg gelangten Burggrafen von Nürnberg aus dem Geschlecht der Hohenzollern ihren neuen Stammsitz errichteten. ${ }^{41}$

Zwischen Sumpf und Sand eine Insel, entstanden im Dazwischen kleiner märkischer Handelsplätze. Anfangs ohne jede Bedeutung, und bedeutungslos auch die Menschen, welche diesen Flecken bewohnten. Da gab es keine Gründung durch das Römische Imperium, ganz zu schweigen von einem Gotte, der einst befohlen hätte, dass hier fortan eine Stadt sei. In L'Ile aux musées wird diese Geschichte wie im Zeitraffer in wenigen Zeilen in den Roman eingeblendet: Stück für Stück habe man die Berliner Insel als Gemüsegarten des Königs, dann als Botanischen Garten genutzt und schließlich - auf königlichen Befehl von 1797 - mit Brücken verbunden und in der Folge als Museumsinsel verwendet. ${ }^{42}$ Ein ehemaliger Gemüsegarten also, wenig heroisch - aber immerhin eine Insel inmitten von Fließgewässern und Sümpfen.

Wenn wir eine Insel als einen allseits von Wasser umgebenen Raum begreifen, der sich durch eine fundamentale infrastrukturelle Diskontinuität - also die Notwendigkeit, beim Erreichen oder Verlassen der Insel das Transportmittel zu wechseln - kennzeichnet, dann stellt sich die Frage, ob wir es im strengen Sinne bei der Berliner Museumsinsel überhaupt mit einer Insel zu tun haben. Die Antwort hierauf fällt aus dieser Perspektivik deutlich aus. Denn noch bevor im 19. Jahrhundert das erste, heute so genannte „Alte Museum“, zwischen 1825 und 1830 auf der Berliner Stadt-Insel errichtet worden war, hatte die künftige

41 Schade, Günter: Die historische Entwicklung der Berliner Museumsinsel. In: Die Museumsinsel zu Berlin. Mit Beiträgen von Peter Betthausen, Anne Effenberger, Volkmar Enderlein, Heinz Fengler, Edith Fründt, Irene Geismeier, Burkhardt Göres, Liane Jakob-Rost, Erika Karasek, Max Kunze, Wolfgang Müller, Siegmar Nahser, Günter Schade, Werner Schade und Eva Zengel. Mit Farbaufnahmen von Dietmar und Marga Riemann. Berlin: Henschelverlag 1987, S. 7.

42 Wajsbrot, Cécile: L’lle aux musées, S. 12. 
Museumsinsel in dem Maße, wie sie von der Peripherie ins Zentrum rückte und über Brücken mit dem Festland verbunden wurde, bereits aufgehört, eine Insel zu sein.

Eine der namenlos bleibenden Protagonistinnen merkt daher zurecht an: „Und ich habe mich auf diese Museumsinsel gestürzt, weil ich Lust hatte, auf einer Insel zu sein, aber es handelt sich um eine urbane Insel, die nichts Insulares besitzt, ist sie doch mit Brücken verbunden, auf denen Autos fahren." “43 Eine Stadt-Insel ist es, die über eine Vielzahl von Brücken und (wie im Falle des Bode- oder des Pergamon-Museums ${ }^{44}$ ) Brückenteilen erreichbar ist - und damit eine paradoxe Stadt-Insel. Doch die Stimmen der Statuen beharren trotzig auf der Insularität dieser „̂̂le urbaine“, von der aus weltweite Bezüge zu anderen Inseln hergestellt werden:

Sie sind auf einer Insel, Sie scheinen dies zu vergessen - wir wissen, dass es weit entfernte, von Erde bedeckte Inseln gibt, Inseln ohne Brücken inmitten des Meeres, und es braucht Schiffe und Schiffstage, um sie zu erreichen, wir wissen, dass manche niemals dorthin gelangen, dass Schiffbrüche passieren. Wir wissen, dass auf bestimmten Inseln kolossale Statuen wohnen, von denen niemand den Ursprung kennt. ${ }^{45}$

Die Statuen auf der Berliner Museumsinsel sind wohlinformiert und wissen Bescheid. Sie wissen von ,echten` Inseln, zu denen keine Brücken führen, sie wissen sogar von Inseln, die von Statuen bewohnt werden, von denen niemand weiß, ob Menschen sie einst erschufen oder ob diese Statuen schon immer da lebten. Sie wissen, dass die Erde nicht nur aus Kontinenten gebildet ist, dass kleine Fetzen Land über riesige Teile der Erde verstreut sind und dass immer wieder Schiffbrüche passieren, welche alle Verbindungen unterbrechen. Doch sie wissen von diesen Verbindungen, die sich zwischen Inseln herstellen, wissen von Archipelen, die miteinander in Verbindung stehen, auch wenn sie auf der anderen Seite der Erde liegen.

Die Vieldimensionalität der Insel als Teil einer weltweit vernetzten Inselwelt, die zugleich als Insel-Welt eine Welt für sich bildet, stellt sich freilich nicht allein auf globaler, sondern auch auf lokaler Ebene. Dabei muss die historische Frage mitbedacht werden, ob es sich bei der Stadtlandschaft Berlins um eine oder um

43 Ebda., S. 38: „et je me suis précipitée dans cette île aux musées parce que j’avais envie d'être sur une île mais c'est une île urbaine qui n'a rien d'insulaire, reliée par des ponts où circulent des voitures."

44 Vgl. hierzu Schade, Günter: Die historische Entwicklung der Berliner Museumsinsel, S. 22 u. 33.

45 Wajsbrot, Cécile: L’Ile aux musées, S. 83. 
viele Inseln handelt, und wo sich - wenn der letztgenannte Fall zutrifft - das ,eigentliche‘ Zentrum befinden könnte:

\begin{abstract}
Unsere Insel wurde den Sümpfen abgewonnen wie die ganze Stadt, die Ablaufgräben wurden trockengelegt, das Terrain eingeebnet, dann wurden Brücken gebaut, um die verschiedenen Teile der Stadt miteinander zu verbinden, was heute als das Zentrum erscheint, war damals weit draußen, und was heute fernab des Zentrums scheint, war die Stadt selbst. Auf unserer Insel haben Sie andere Inseln gebaut, denn die Museen sind eine Art Klöster, in welchen Sie die Geschichte der Zeit einsperren. ${ }^{46}$
\end{abstract}

Die einst von Wilhelm von Humboldt, Karl Friedrich Schinkel und Aloys Hirt, aber auch von den Bildhauern Gottfried Schadow und Christian Daniel Rauch ersonnene ${ }^{47}$ und über ein Jahrhundert lang ständig erweiterte und umgestaltete Insel entfaltet auf engstem Raum eine Kette von Museums-Inseln, die wie eine Inselkette der Kulturen den kulturellen Brückenschlag von Berlin aus zu den Weltkulturen herstellt. Die Berliner Museumsinsel zeigt sich uns so als eine „InselInsel“, auf der weitere Inseln beherbergt sind, welche mit Inseln der ganzen Welt in Verbindung stehen. Denn die „Ile aux musées“ ist durch archipelische und transarchipelische Bezüge mit der gesamten 'Erde verknüpft.

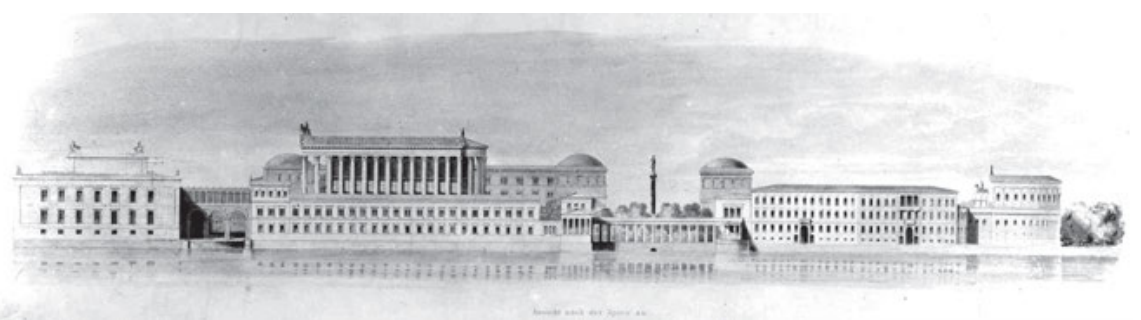

Abb. 134: Gesamtplan der Museumsinsel, Aufrisszeichnung von Friedrich August Stüler, 1841.

Auf Grundlage der inter- und transkulturellen Relationalität der Statuen, die den unterschiedlichsten Zeiten und Räumen, Gesellschaften und Kulturen entstammen, generiert sich dieser Brückenschlag in Cécile Wajsbrots Roman auf eine Weise, welche die nicht nur museale Zukunft in der Mitte Berlins geradezu vorauszusehen und vorwegzunehmen sucht. Denn schon in naher Zukunft soll sich das gegenüber der (zum Weltkulturerbe der Menschheit zählenden) Museumsinsel liegende und mit einem neuen Intendanten wieder flott gemachte Hum-

46 Ebda., S. 84.

47 Vgl. Schade, Günter: Die historische Entwicklung der Berliner Museumsinsel, S. 12f. 


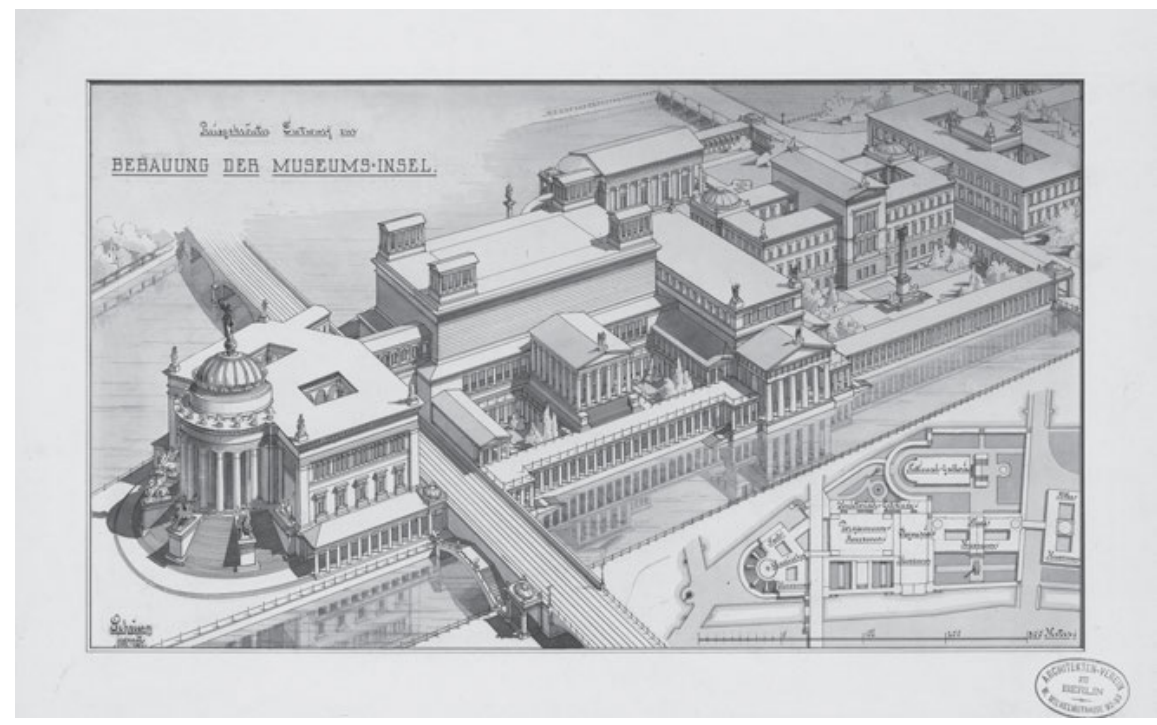

Abb. 135: Gesamtanlage für die Erweiterung der Museumsinsel, perspektivische Ansicht von Bernhard Sehring, 1882.

Ort aus intensiviert wird, der sich nicht länger als das Zentrum, wohl aber als eine in besonderer Weise konzipierte Spielfläche kultureller Inselwelten versteht? Endgültig vorüber - wenn auch architektonisch noch gegenwärtig - ist die Zeit, in der sich große Ausgrabungsprojekte mit imperialen Herrschaftsansprüchen verbanden, um nach Gründung des Deutschen Reiches dessen Größe, aber auch dessen expansionistische Ansprüche in der Mitte seiner Hauptstadt zu dokumentieren und gebührend in Szene zu setzen. Wir leben heute glücklicherweise in anderen Zeiten; und es wird ganz wesentlich darum gehen, die Museumsinsel nicht in ein Zentrum, sondern einen Knotenpunkt inter- und transkultureller Beziehungen und eines viellogischen, archipelischen und transarchipelischen Verständnisses der Kulturen der Welt zu verwandeln.

Cécile Wajsbrots zweiter Roman innerhalb ihres romanesken Zyklus eröffnet den Raum, in dem sich die Gestalten der Friese des Pergamon-Altars mit Skulpturen austauschen, welche die Erfahrungen des Zweiten Weltkriegs reflektieren und so eine transhistorische Welt der Statuen konfigurieren, in welcher Der Rufer, „Celui qui appelle“, dessen unhörbares Schreien den Romanauftakt bildet, viele Hörerinnen und Hörer, zuvor unvermutete Gesprächspartner gefunden hat. Denn scheint es nicht dieser Rufer gewesen zu sein, der - im Frühjahr 1989 aufgestellt wenige Monate später die graue Berliner Mauer gleichsam zum Einsturz brachte? So heißt es am Ende des kurzen Auftaktkapitels: „Sechs Monate nach der Instal- 
lierung des ewig unbeweglichen Mannes mit dem ewig geöffneten Mund fiel die Berliner Mauer.“48

Als Inselbrücke der Kulturen, die aus unterschiedlichsten Inseln hervorgegangen ist, mit den verschiedenartigsten Inseln weltweit in Verbindung steht und auf ihrem Boden höchst differente Museums-Inseln mit ihrer Eigen-Zeitlichkeit beherbergt, bildet Cécile Wajsbrots L'Ile aux musées ein geradezu perfektes literarisches und kulturelles Fraktal. In dessen Selbstähnlichkeit verbergen und enthüllen sich zugleich unter der einen Insel stets andere Inseln, unter der einen Geschichte stets andere Geschichten, unter der einen Stadt stets andere Städte. Die Berliner Museumsinsel verwandelt sich unter der Feder der französischen Autorin in ein vollendetes WeltFraktal.

Es verwundert daher nicht, dass in einer Anspielung auf ,ces mathématiciens qui disent que la côte du Finistère est infinie“49 - also auf Mathematiker, welche die Unendlichkeit der nördlichen Küsten Frankreichs berechnen - im zentralen Teil des Romans der (freilich namentlich nicht genannte) Erfinder einer fraktalen Geometrie der Natur eingeblendet wird. ${ }^{50}$ Die offene, viellogische Strukturierung, die sich in L'Ile aux musées auf ästhetisch wie ethisch beeindruckende Weise verdichtet, bündelt in Form einer fraktalen Insel der Inseln Geschichte(n) schlechthin. Dies bedeutet in der Tat, dass die Insel zum Modell für eine fraktale Konzeption weltweiter kultureller Relationen geworden ist, welche sich wohl in Form des Archipels am besten konfigurieren lassen.

Keine Geschichte, kein Ort, nichts, so scheint uns der Roman zu suggerieren, geht auf dieser Insel aller Inseln jemals verloren. Das Abwesende, da Zerstörte, ist in und dank seiner Absenz präsent. In Cécile Wajsbrots Ästhetik der Abwesenheit, ${ }^{51}$ in der das Vergangene - wie David im Titel ihres Romans Caspar-Friedrich-Strasse - gerade durch die Lücke, die Verrückung, die Vernichtung präsent wird, sind die nicht sichtbaren Inseln unter den Inseln, die verborgenen Städte unter den Städten immer ins Bewusstsein, ins Weltbewusstsein gehoben. Von den Kriegen Alexanders des Großen bis zum Kalten Krieg, von der (gleich zu Beginn

48 Wajsbrot, Cécile: L’Ile aux musées, S. 9: „Six mois après l'installation de l'homme éternellement immobile à la bouche éternellement ouverte, le mur de Berlin tombait.“

49 Ebda., S. 106.

50 Vgl. Mandelbrot, Benoît B.: Die fraktale Geometrie der Natur. Aus dem Englischen übersetzt von Dr. Reinhilt Zähle und Dr. Ulrich Zähle. Basel - Boston - Berlin: Birkhäuser Verlag 1991.

51 Vgl. hierzu Ette, Ottmar: „Caspar-David-Friedrich-Strasse“. Cécile Wajsbrot oder die Ästhetik der Abwesenheit. In: Böhm, Roswitha / Zimmermann, Margarete (Hg.): Du silence à la voix Studien zum Werk von Cécile Wajsbrot. Göttingen: V\&R unipress 2010, S. 223-239; vgl. auch das Cécile Wajsbrots Romanschaffen gewidmeten achte Kapitel in (ders.): ZwischenWeltenSchreiben. Literaturen ohne festen Wohnsitz, S. 239-263 sowie S. 54-59. 
des Romans eingespielten) Eroberung Trojas bis zum Ausbruch des Irak- wie des Afghanistan-Krieges ist in ihnen - wie in der Literatur - alles in den jeweiligen Bewegungsfiguren vektoriell gespeicherte Präsenz, weit jenseits des alltäglichen Präsens der Menschen. Die Insel wird zur Chiffre der Komplexität aller Lebensverhältnisse und der Beziehungen zwischen den Kulturen, welche auf ihr gelebt und erlebt werden können.

So entfaltet Cécile Wajsbrots Roman nicht nur eine Archäologie, die in gleichsam vertikaler Richtung Schicht um Schicht der Geschichte freilegt, sondern auch eine dynamische Kartographie der Bewegungen, die in horizontaler Richtung die Deportationen und Migrationen verzeichnet, die an einem Ort immer den anderen Ort präsentieren, ja jegliche Repräsentation eines Ortes stets mit dessen AnderOrten vernetzen. Keine Raumgeschichte, sondern nur eine Bewegungsgeschichte kann diese sich weiter beschleunigende Fülle an De- und Reterritorialisierungen noch erfassen. Wir müssen uns auf den Roman, auf sein dichtes Geflecht an nur auf den ersten Blick versteinerten Blicken einlassen, um all dies bewegungsgeschichtlich zu erleben und durchzuerleben.

Nicht nur mit Bezug auf die so andere und zugleich doch vielverbundene Pariser Stadt-Insel der Ile de la Cité lässt sich von der Museumsinsel in Berlin sagen: „Une île peut en cacher une autre.“ Wie in Emine Sevgi Özdamars Romantrilogie erscheint bei Cécile Wajsbrot unter der Oberfläche Berlins nicht Istanbul, aber Paris, tauchen im Herzen von Paris die Strukturen der Stadt an der Spree auf. Paris und Berlin werden nicht miteinander identisch, sie verschmelzen miteinander nicht, sind jedoch voneinander nicht mehr trennbar, sondern überlagern sich entlang immer neuer Blickachsen: Die französische und die deutsche Hauptstadt verwachsen bei der zwischen Paris und Berlin pendelnden französischen Schriftstellerin zu „Parlin“.

Eine Stadt ist nicht nur ihre Geschichte vor Ort, ihre Tiefe, sondern auch ihre Vektorizität, ihre mobile, dynamische Vielverbundenheit in der Fläche, im transarealen Maßstab. Die viellogischen Strukturen dieser Stadt unter der Stadt machen auf Ebene ihrer unterschiedlichen Museen und Kunstwerke die offene Strukturierung eines weltumspannenden Archipels aus, in dessen lebendigen Kartographien die Choreographien samt all ihrer Zerstörungen, all ihrer Abwesenheiten eingetragen sind. Ist das Museum nicht jener Bewegungs-Raum, an dem das Sammeln an vielen Orten in die Sammlung an einem Ort übergeht? Könnten wir den Raum eines Museums jemals verstehen, würden wir in ihm allein seine statische, architektonische Spatialität und nicht die Generierung dieses Raumes durch die ihn kreuzenden Bewegungen berücksichtigen? Ist das Museum nicht der Ort, an dem wir durch das Geflecht von Blicken zwischen den Objekten, zwischen den Betrachter*innen und den Objekten sowie zwischen den Besucher`innen untereinander jene notwendig bewegungsgeschichtliche 
Dimension erfahren können, wie sie uns in der Kunst etwa ein Mobile vor Augen führt?

Es scheint nur folgerichtig, wenn sich die nicht in eine simple Gegenwart, sondern in die gegenüber allen Vergangenheiten wie Zukünften geöffnete Präsenz geholten Statuen, deren steinernes Gedächtnis alles aufbewahrt, ihrerseits als Inseln begreifen: „Nous sommes des îles aussi - pour accéder à nous, il faut nous aborder." ${ }^{52}$ Auch die Statuen selbst sind folglich Inseln. Besitzt jede einzelne dieser Skulpturen nicht ihre eigene Logik, ihre eigene Geschichte, ihre eigenen Bewegungsfiguren, die sie weniger aus ihrer Territorialität, ihrer Herkunft, als aus ihrer Vektorizität, ihrer Bewegungsgeschichte heraus begreifbar macht? Der Burg-Berg von Pergamon und die Museumsinsel in Berlin sind transareal unauflöslich miteinander verwoben. Das Alte Museum ist ohne den älteren Louvre nicht vorstellbar. Ein Ort ist viele Orte, und viele Orte verdichten sich an einem Ort zu einem Ort.

Als Großstadtroman macht L'Ile aux musées deutlich: Um eine Stadt wie Berlin $\mathrm{zu}$ begreifen und literarisch $\mathrm{zu}$ inszenieren, genügt es nicht, ihre Archäologie, ihr Gewachsen-Sein an Ort und Stelle, mit den Mitteln von Geschichte und Literatur darzustellen. Entscheidend kommt es vielmehr darauf an, ihre Vektorizität, ihre historisch akkumulierten und dadurch spezifisch in Gegenwart und Zukunft projizierten Bewegungsmuster zu entfalten, um die Stadt selbst als ein komplexes, sich ständig veränderndes Bewegungsbild zu verstehen. Eine Stadt besitzt keine Wurzeln (,roots“), sondern entsteht aus Wegen („routes“). Sie ist wie das menschliche Leben aus Bewegungen, aus Migrationen gemacht: Allein in ihrer akademischen Abstraktion entsteht ihr statisches Bild, das wir in Leben verwandeln müssen.

Dabei geht es nicht nur um Relationalität und die mit ihr verbundenen Bewegungen, sondern um unaufhörliche Überlappungen und Überblendungen im eigentlichen Sinne: In und unter der einen Stadt wird immer eine andere sichtbar, lebbar und erlebbar, so wie ohne die Einbeziehung der je anderen Stadt die eigene letztlich unbegreifbar und unverstanden bleiben muss. Eine Stadt schafft sich ihr eigenes Bild, indem sie als Fraktal möglichst viele Städte in sich in Bewegung setzt: Berlin beinhaltet Istanbul wie Paris; und in Paris und Istanbul stoßen wir auf Berlin.

Es dürfte wohl nicht nur die autobiographische Erfahrung der zwischen Paris und Berlin pendelnden Autorin gewesen sein, die in Cécile Wajsbrots Roman im Zeichen der deutsch-französischen Beziehungen eine besonders verdichtete Relationalität zwischen den urbanen Räumen der französischen und deutschen

52 Wajsbrot, Cécile: L’Ile aux musées, S. 85. 
Hauptstadt geschaffen hat. In L'Ile aux musées wird der titelgebende Ort genau dadurch konfiguriert, dass ihm in ständigen Überlappungen und Überblendungen die Insel im Herzen von Paris, die Ile de la Cité, gemeinsam mit dem Louvre an die Seite gestellt wird. Sie ist ganz anders strukturiert als die Berliner Museumsinsel. Daher lässt die Tatsache, dass es sich um eine von Menschen sehr dicht bewohnte und keineswegs in eine Kette von Museen aufgespaltene, aber doch wiederum in eine museale Stadtlandschaft eingebundene und von den Blicken der Statuen mit dem Festland verwobene Insel handelt, eine vielfache Spiegelung insulärer Strukturen zu, welche die deutsch-französischen Gegenmodelle in eine wechselseitige Komplementarität einbinden. Hier wird die Fähigkeit geschult und ist dies nicht die eigentliche Aufgabe der Literatur? -, in unterschiedlichen Logiken zugleich $\mathrm{zu}$ denken und ein viellogisches Weltbewusstsein $\mathrm{zu}$ entfalten.

Die so bestimmte Komplementarität dient nicht nur den stets aufeinander verweisenden Städten Paris und Berlin als immer wieder anders gespiegeltes Raum- und Denkmodell. Denn es erfüllt diese Funktion ebenso für die Statuen, die zwischen Berlin und Paris miteinander in einen dichten Dialog eintreten, wie für die beiden namenlosen Liebespaare, die sich wie Fragmente eines Diskurses der Liebe flüchtig konfigurieren und zwischen beiden Städten finden und wieder verlieren. In den Zwischenräumen der mobilen Kartographien, die sich zwischen den sich überlagernden Städten und Stadtinseln von Paris und Berlin herausbilden, entstehen zögernd vorgebrachte Fragmente einer Sprache der Liebe, die sich im Zeichen der Kunst, im mehrfachen Sinne unter dem Blick der Statuen aus Marmor, Granit oder Bronze, eher flüchtig (also ephemer und wie auf der Flucht) entfalten.

Das all diesen menschlichen und allzumenschlichen Beziehungen aufgeprägte Signum ist das „Panta Rhei“, das am Ende, im Excipit des Romans, ein letztes Mal mit Blick auf eine der anonymen Liebesfiguren eingespielt wird, die sich schon bald in der verdoppelten Stadtlandschaft von Parlin verliert:

Sie hat die zentrale Allee wieder erreicht und wird Teil des Fließens der Spaziergänger, die zum Tore laufen, als ob sie dem Verlaufe eines Flusses folgten. Eines der Tore ist geschlossen, das andere steht noch offen. Ein Wächter wartet, bis der Garten sich leert, um ihn dann wirklich abzuschließen. Aus der Ferne unterscheiden wir sie kaum noch von den anderen, aus der Ferne sind es nur Silhouetten. Sie ist gegangen, alle sind gegangen. Das Tor schließt sich wieder. Und wir sind da. Wir bleiben. ${ }^{53}$

53 Wajsbrot, Cécile: L’lle aux musées, S. $229 \mathrm{f}$. 
Was sich im Verlauf des Romans, im Verlauf zufälliger Begegnungen und geplanter Museums- und Ausstellungsbesuche „en filigrane“ als Gewebe der Gefühle entfaltet, speist sich am Ende, wenn der Garten als Spielfläche für all diese Gefühle erzählerisch präzise geschlossen wird, wieder ein in den Fluss, in das „Alles fließt“ aller von Menschen ersonnenen, empfundenen und erlebten Dinge. Dies geschieht allein, um wie das Wasser am Horizont einer langlebigen Kunst zu entschwinden. Die Menschen entfernen sich, werden zu Silhouetten, verschwinden am Horizont. Die Statuen aber bleiben, das Werk der Künstler und der Kunst bleibt und überdauert die Menschen.

Was aber bleibt, wenn sich das Abwesende durch seine Abwesenheit als Anwesendes zeigt? Das Gewebe der Blicke und Gefühle geht im Gewebe des von der Kunst erzeugten Erlebens auf, wird im doppelten Sinne aufgehoben in einer Kunst der Literatur, die den Verheerungen menschlicher Geschichte(n) nie gänzlich entzogen und doch niemals auf sie zurückgeworfen, reduzierbar ist. Das Leben der Menschen ist prekär, ist ephemer: Es fließt und fließt vorbei. L'Ile aux musées legt - wie die künftige Berliner Museumsinsel mit ihrem Schlossareal des Humboldt-Forums - von diesem Wissen, von diesem Lebens- und Zusammenlebenswissen, ein ebenso faszinierendes wie dauerhaftes Zeugnis ab: in Zeit und Raum verankert, doch in den kunstvoll geschaffenen erlebbaren Wirklichkeiten weit über die Zeit und die Menschen und ihr Präsens hinausragend.

So entfalten die mobilen Kartographien urbaner Räume im ZwischenWeltenSchreiben von „Berlistan“ und „Parlin“ ein Reich der Zeichen, das aus der langen Tradition der Beziehungen zwischen Urbanität und Literatur zu schöpfen vermag, um vom urbanen Bewegungs-Raum aus ein Bewusstsein von der Welt zu entfalten. Letzteres versteht es, aus dem Mikrokosmos der Stadt, dem Mikrokosmos der Literatur alle Zeiten und alle Räume an einem Ort - wie in einem borgesianischen „Aleph“ - zu verdichten. Diese Dimensionen urbaner Stadtlandschaften sind auch in Cécile Wajsbrots Roman Destruction gegenwärtig, der im Jahre 2019 ihren Zyklus Haute Mer krönte.

Denn in diesem Abschlussroman fließen die insistierenden Bilder des gesamten Zyklus auf großartig verdichtete Weise zusammen und überlagern sich eindringlich in ihren Isotopien des Suchens und Sich-Verfehlens, der ständigen Bewegungen zwischen den Städten Paris und Berlin oder der Metaphorik von Sonnenfinsternis und Soleil noir. ${ }^{54}$ Destruction vereinigt diese Bilder und Bedeutungsebenen in einem literarischen Entwurf von Störung, Zerstörung und Selbstzerstörung, in welchem der zunächst unmerkliche Übergang einer Demokratie zur Diktatur zentral gestellt ist. Das Thema der Katastrophe und des Verschwindens 
der Menschheit, das in verschiedenen Romanen des Zyklus durchgeführt wird, taucht so in einer Form wieder auf, welche den beunruhigenden Aufstieg der Populisten und Rechtsradikalen sowie den Wiederaufstieg von Autoritarismen jeglicher Couleur bis hin zur Selbstzerstörung der Menschheit reflektiert. Denn was zunächst als Störung begann, endet in der Zerstörung und Selbstzerstörung eines demokratisch verfassten Gemeinwesens, mit dem eine ganze Epoche zu Ende zu gehen scheint.

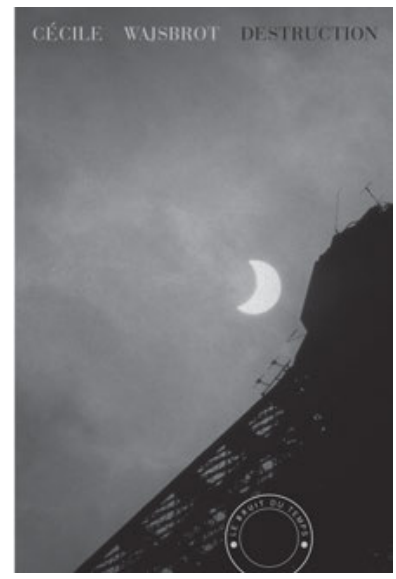

Abb. 136: Cécile Wajsbrot: Destruction (Cover, 2019).

$\mathrm{Zu}$ Beginn des Romans noch gilt ein Rückfall in die politischen, militärischen und menschlichen Katastrophen des 20. Jahrhunderts als ausgeschlossen. Es ist eine andere, optimistisch nach vorn blickende Zeit, die zu Beginn eines neuen Jahrtausends im Bewusstsein einer weiter vorantreibenden Beschleunigung der Globalisierung die aufziehenden und für die Menschheit gravierenden Probleme nicht kommen sieht. Die Ich-Erzählerin wusste sich lange Zeit mit ihrer Generation vereint in dem Glauben, keinen Rücksturz mehr in das Jahrhundert der Kriege, Katastrophen und massiven Migrationen erleben zu müssen, da man nunmehr im Zeichen von Wissenschaft und Fortschritt diese Vergangenheit mörderischer Diktaturen ein für alle Mal hinter sich gelassen habe. Es ist der Rückblick auf eine Epoche, von der selbst schwergewichtige deutsche Politiker sagen, sie hätten an die Entstehung einer neuen, gerechteren Weltordnung und nach Ende des Kalten Krieges an das Heraufziehen eines ewigen Weltfriedens geglaubt:

Ich erinnere mich, dass wir an die Zahlen glaubten, die der Mathematik, die der Ökonomie. Wir glaubten, dass sie die Welt regierten, dass es genügen würde, sie zu kennen - oder wage ich zu sagen, sie zu dechiffrieren, sie zu erzählen? Wir betrachteten sie als eine Art Gottheit - ein wenig wie die Mayas, die jede Zahl mit einem Gott verbanden -, vergaßen dabei 


\begin{abstract}
aber, das wir selbst sie geschaffen hatten. Wir dachten, dass wir mit ihrer Hilfe die Welt regulieren könnten, Regeln und Gesetze, ja Zyklen erkennen würden, die Länder regieren könnten. Dank der Zahlen lebten einige besser als andere, und ich gehörte dazu. Wir glaubten, dass die Zukunft und die Zahlen miteinander verbunden seien. Das einundzwanzigste Jahrhundert würde, so dachten wir, das des Fortschritts, der Wissenschaft, der Eroberung des Weltraumes sein, die das zwanzigste begonnen und gleichzeitig gestoppt hatte. Wir dachten, die Seite der Katastrophen des vorigen Jahrhunderts umgeblättert zu haben. Trotz einiger Zeichen - Flugzeuge, die unerklärlicherweise abstürzten, Stürme von ungekannter Stärke, neue Epidemien - dachten wir, das Schlimmste überwunden zu haben. ${ }^{55}$
\end{abstract}

Hier also die Retrospektive einer Epoche, welche alle grundlegenden Probleme der Menschheit überwiegend gelöst zu haben glaubte. Die Berechenbarkeit der Zyklen der Natur, aber auch jener der Ökonomie schien gewiss, die Kalkulierbarkeit aller Phänomene des Lebens gesichert, ja die Vorhersehbarkeit einer Zukunft hochwahrscheinlich, welche selbstverständlich keinerlei Rückfall in ein Jahrhundert der Katastrophen, Verfolgungen und Unterdrückungen kennen würde. Man fühlte sich sicher und aufgehoben, auch wenn es bisweilen verstörende Zeichen gab wie um sich greifende Epidemien oder in ihrer Gewalt immer stärker ansteigende Wirbelstürme. Auch dies schien letztlich eine Frage von Kalkulierbarkeit und effizienten Gegenmaßnahmen zu sein, welche rechtzeitig eingeleitet werden müssten. Dann würde schon alles gut gehen ...

Doch recht rasch begreift man im weiteren Fortgang der Lektüre, dass der abstrakte Gedanke an die ein für alle Mal abgeschlossene Vergangenheit der Diktaturen mittlerweile der Erfahrung konkreter Möglichkeiten ihrer Wiederkehr Platz gemacht hat. Längst hat - im Umfeld von Wahlen in Frankreich, die nicht präzise zeitlich eingeordnet werden - der Übergang in eine autoritäre Herrschaft eingesetzt, der alle vorherigen Gewissheiten der Ich-Erzählerin und ihrer Generation zerstört. Die Katastrophen der Kultur, der Regierung und Regierbarkeit treten in den Vordergrund der Romanhandlung. In diesem Zusammenhang wird deutlich, dass Vergangenheit und Gegenwart nicht fein säuberlich voneinander getrennte oder überhaupt trennbare Einheiten darstellen, sondern dass beide Zeitebenen auf intime Weise miteinander verbunden sind: „Wie ziehen Sie eine dichte Grenze zwischen Gegenwart und Vergangenheit? Ich spreche in der Gegenwart zu Ihnen, gewiss, doch es gibt Bilder, die mich verfolgen, die mich heimsuchen, Bilder vom Leben zuvor. “56 Das vergangene Leben ist im gegenwärtigen noch immer gegen-

55 Wajsbrot, Cécile: Destruction, S. 32.

56 Ebda., S. 51: „Comment tracez-vous une frontière étanche entre le présent et le passé? Je vous parle maintenant, bien sûr, mais des images me hantent, viennent me visiter, celles de la vie d'avant.“ 
wärtig als eine Vergangenheit, die nicht enden kann und nicht enden will. So wie sich im Raum die Räume überlagern, so wie in der einen Stadt die andere präsent ist, so überlagern sich auch die Zeitebenen, durchdringen einander, werden nicht identisch, verschmelzen nicht miteinander, aber sind doch untrennbar liiert und miteinander verbunden.

Wie auf der individuellen Ebene die Bilder der Vergangenheit ständig in der Gegenwart präsent und präsentisch sind, gilt eine derartige Vergegenwärtigung auch auf kollektiver Ebene, jener einer „mémoire collective récente“, ${ }^{57}$ einer aktuellen kollektiven Erinnerung, in welcher die Bilder früherer Katastrophen sehr wohl in einem kollektiven Erinnerungsraum gespeichert bleiben. Dabei verweist der hier verwendete Begriff der „mémoire collective“ auf seinen Schöpfer Maurice Halbwachs ${ }^{58}$ und damit über dessen Tod im Konzentrationslager Buchenwald hinaus auf die Katastrophe der Nazi-Barbarei und der Shoah selbst.

Das Thema der Judenverfolgung, der Judenvernichtung, ist in allen Werken Cécile Wajsbrots präsent: Es bildet eine Vergangenheit, deren Bilder die Autorin immer wieder heimsuchen. Gerade die Grenzen zwischen Vergangenheit und Gegenwart, aber auch zwischen beiden und der Zukunft sind weder statisch noch undurchlässig, sondern beruhen - entgegen der Überzeugung, im Zahlen- und Fortschrittsoptimismus die Zukunft genau berechnen zu können - auf ständigen Wechselwirkungen und Projektionen. Wir haben es hier nicht mit eindeutigen Grenzlinien, sondern mit widerspruchsvollen Spannungsfeldern zu tun. Die Bilder der Vergangenheit sind in der Gegenwart präsent, sind Gegenwart und hören auch nie auf, Gegen-Stand der Gegen-Wart zu sein.

Von den allerersten Zeilen des literarischen Schlusssteins des Romanzyklus Haute Mer an, ist das sicherlich zentral gestellte Bild der Sonnenfinsternis allgegenwärtig, mit dem sich die französische Autorin bereits im vierten, also unmittelbar vorangehenden Roman Totale éclipse intensiv auseinandergesetzt hatte. In Destruction wird dabei nicht nur auf die exakte zahlenmäßige Berechenbarkeit von Sonnen- oder Mondfinsternissen hingewiesen, sondern auch auf die Tatsache aufmerksam gemacht, dass diese Konstellationen in unserem Planetensystem symbolische Bedeutungsebenen transportieren, welche durch ihre astronomische Kalkulierbarkeit keineswegs rational auszublenden sind. Wie Vergangenheit und Gegenwart sind auch Realität und Fiktion nicht voneinander zu trennen. Fiktionen sind Teile unserer Wirklichkeit. Und Sonnenfinsternisse verheißen in allen Menschheitskulturen nichts Gutes.

57 Ebda., S. 157.

58 Vgl. die neuere Ausgabe von Halbwachs, Maurice: La mémoire collective. Paris: Albin Michel 1997. 
Eine besondere Bedeutungsebene wird in den Roman aber durch den Hinweis darauf eingeblendet, wie unmerklich diese Vorgänge stets einsetzen und wie schwer wahrnehmbar der Beginn einer Sonnenfinsternis ist, selbst wenn wir den Eintritt der Scheibe des Mondes auf die noch sichtbare Sonnenoberfläche genau vorherbestimmen können. Der Aspekt des Unmerklichen wird in den Vordergrund gerückt:

\begin{abstract}
Vor etwa zwanzig Jahren waren wir an den höchsten Punkten von Paris Hunderte, die den Himmel absuchten in Erwartung des Augenblicks, an welchem der Schatten endlich erscheinen würde, unmerklich erst, dann größer werdend. Es war mitten in der Stadt - und der Blick über Paris ging über die einzelnen Flächen der Dächer, auf einem urbanen Hintergrund mit wohlbekannten Formen, die sich voneinander abhoben, die Farben von Beaubourg, die regelmäßigen Türme einiger Kirchen, die Wölbung der Bahnhöfe, das Bild einer konstruierten und friedlichen Hauptstadt, die das Wesentliche ihrer Arbeit verrichtet hatte und die sich jetzt ausruhen und von ihren Renten leben konnte. Wir drehten den Rücken der Kirche, der Basilika zu, die zum Zeichen der Reue nach dem Bürgerkrieg, der den Namen der Commune trägt, in Paris errichtet wurde als Symbol einer Buße, welche das Denken von anderen Regimen zu anderen Zeiten und bis heute vielleicht nährte, jetzt aber in eine Touristenattraktion verwandelt ist. Ich wusste damals nicht, dass der Basilika eine Abtei vorausging, die den Bedrängten und Verfolgten als Zufluchtsort gedient hatte, während Sacré Coeur - denn so lautete ihr Name - nur für jene schlug, die ihre Sichtweise der Dinge teilten ... Den Rücken diesem Gebäude zugewandt, schauten wir gen Himmel in der Erwartung des feierlichen Augenblicks. Es war das letzte Jahr des Jahrhunderts, bald sollten wir in die jetzige Ära eintreten - selbst wenn die Welt, in welcher wir seitdem leben, nicht mehr allzu viel gemein hat mit der vorherigen Welt. Doch es war nicht - noch nicht - der Wechsel des Jahrhunderts, auf den wir an jenem Tage warteten, es war am Himmel, wo sich alles abspielen sollte, denn der Lauf der Planeten sollte mit dem des Mondes zusammenfallen und exakt seinen Schatten auf die Oberfläche der Sonne werfen.

Es war eine recht zahlreiche Gruppe, die mehrfach den falschen Alarm oder Wolken kommentierte, welche die Sonne zu verhüllen drohten, die beim Erscheinen pfiffen, als wären sie bei einem Fußballspiel. Ich frage mich, ob nicht sie es schon waren, die die Zerstörung brachten. ${ }^{59}$
\end{abstract}

Die „Totale éclipse“ dieser in Teilen West- und Mitteleuropas fleißig beobachteten Sonnenfinsternis vom 11. August 1999 weist buchstäblich mit ihren Kernschatten voraus auf ein neues Jahrhundert und vielleicht sogar Jahrtausend. Die Erzählerin lässt keinen Zweifel daran aufkommen, dass dies eine neue Ära ist, für welche die Zeichen dieser allgemeinen Verfinsterung symbolhaft stehen. Die große Gruppe von Parisern und Touristen, welche des epochalen Augenblicks in keiner Weise gewahr sind, ahnt nichts von der symbolischen Wende und dreht ganz bewusst die Erzählerin betont es zweifach - der Vergangenheit den Rücken zu, einer

59 Wajsbrot, Cécile: Destruction, S. $65 \mathrm{f}$. 
blutigen Vergangenheit, die in den Morden an den Communards gipfelte und sich dazu der Hilfe eines Kirchenbaues bediente. Doch all dies scheint für immer beendet und abgeschlossen zu sein: „on a tourné la page.“

Das Vorrücken des Dunklen auf der Oberfläche des Lichts wiederholt sich auf vielen Ebenen des Romans, der wie ein Schlussstein den Bogen eines Rückblicks auf das 20. Jahrhundert krönt. Destruction gibt am Ende des Romanzyklus zugleich den Blick auf eine Zukunft frei, die entgegen aller anfänglichen Hoffnungen wohl nicht unbedingt mehr Licht in das Dunkel einer Menschheitsgeschichte $\mathrm{zu}$ bringen verspricht, welche noch immer im Schatten des vergangenen Jahrhunderts steht. Denn die Katastrophen dieses vergangenen Jahrhunderts sind nur scheinbar vergangen: In Wirklichkeit sind sie inmitten der Gegenwart präsent und werfen für die Zukunft düster ihre Schatten voraus.

Alle Zeichen werden zu möglichen Vor-Zeichen des Künftigen: So wie Walter Benjamins Engel der Geschichte in seinem Vorwärtsgetrieben-Sein durch den Sturm vom Paradies her auf die Überreste all jener Katastrophen blickt, die sich auf seinem Weg angehäuft haben. Denn im Rückgriff auf ein „Bild von Klee, das Angelus Novus heißt“ ${ }^{60}$ hielt Walter Benjamin eindrücklich fest:

Der Engel der Geschichte muß so aussehen. Er hat das Antlitz der Vergangenheit zugewendet. Wo eine Kette von Begebenheiten vor uns erscheint, da sieht er eine einzige Katastrophe, die unablässig Trümmer auf Trümmer häuft und sie ihm vor die Füße schleudert. Er möchte wohl verweilen, die Toten wecken und das Zerschlagene zusammenfügen. Aber ein Sturm weht vom Paradiese her, der sich in seinen Flügeln verfangen hat und so stark ist, dass der Engel sie nicht mehr schließen kann. Dieser Sturm treibt ihn unaufhaltsam in die Zukunft, der er den Rücken kehrt, während der Trümmerhaufen vor ihm zum Himmel wächst. Das was wir den Fortschritt nennen, ist dieser Sturm. ${ }^{61}$

So wie die Kriege, Konflikte und Katastrophen des 19. und 20. Jahrhunderts ganzim Sinne Benjamins in Cécile Wajsbrots Romanzyklus allgegenwärtig sind, so zeigen sich in Destruction auch immer wieder Bilderfolgen, in denen sich unmerklich die kommenden Katastrophen andeuten und abzeichnen. Immer größere, vom Menschen verursachte Naturkatastrophen ereignen sich, unbekannte Epidemien überqueren die Kontinente, das Irrationale mit all seinen Begleiterscheinungen hält Einzug. Dunkle Vögel belagern die Stadt und nehmen sie ein: „Die Raben besetzen die Stadt. Sie sind Zug um Zug mit den Jahren gekommen. Unmerk-

60 Benjamin, Walter: Über den Begriff der Geschichte. In (ders.): Gesammelte Schriften. Band I, 2. Herausgegeben von Rolf Tiedemann und Hermann Schweppenhäuser. Frankfurt am Main: Suhrkamp 1980, S. 695.

61 Ebda., S. $697 \mathrm{f}$. 
lich.“62 Erneut liegt hier der Schwerpunkt auf dem Unmerklichen, der menschlichen Wahrnehmung nicht Zugänglichen. Wer vermag zu erkennen, wann alles begonnen hat, wann die Katastrophe begann? Wann fing alles an, in Zerstörung und Selbstzerstörung überzugehen?

Schritt für Schritt dringen Überwachungs- und Unterdrückungsstrukturen den Raben ähnlich - in Cécile Wajsbrots Roman in die Strukturen des lange Zeit demokratischen Gemeinwesens ein und verändern es grundlegend. An verschiedenartigsten Grenzen zwischen Demokratie und Diktatur bemerken wir, wie Übersetzungsprozesse aus dem einen ins andere Regime, aus dem einen ins andere Jahrhundert, vorgenommen werden und die vorhandenen, noch demokratischen Strukturen zerstören. Zunächst versucht man, die Vergangenheit zu kontrollieren, indem man ihre Zeugnisse ebenso vernichtet wie materielle Träger des Vergangenen auf individueller, familiärer oder kollektiver Ebene. Mit Waffengewalt werden die Bücher aus den öffentlichen wie privaten Bibliotheken entfernt; Familienalben werden konfisziert und zerstört. Es handelt sich um Vorgänge, die wir im Verlauf der Geschichte an verschiedensten Ecken unseres Kontinents und Planeten beobachten konnten, ebenso bei jener von den Nazis monströs und detailliert geplanten Vernichtung der Juden in Europa wie beim Genozid an indigenen Völkern auf dem amerikanischen Kontinent.

Aber auch Bildungsinstitutionen unterschiedlichster Art, von Schulen über Bibliotheken bis hin zu Museen oder Opern, werden umstrukturiert und ihrer Inhalte, ihres Sinns beraubt. Dabei bedient man sich bisweilen populistischer Verfahren und lässt darüber abstimmen, ${ }^{63}$ dass die Kenntnis der Geschichte weniger wichtig sei als die der Geographie, da man schließlich vor allem wissen müsse, wo man lebe. Ebenso wird per Votum festgestellt, dass die Beschäftigung mit der Kunst ein Luxus sei, insofern Operationen für das Volk wichtiger seien als derlei künstlerische Beschäftigungen; oder dass die Restaurierung historischer Gebäude weniger wichtig sei als das Vorantreiben eines sozialen Wohnungsbauprogramms, für welches identische Wohnblöcke in schlechtester Qualität aus dem Boden gestampft werden.

Wir kennen derartige Abstimmungen aus der Geschichte unserer Gegenwart, selbst in Europa, selbst in der Europäischen Union, wo das Volk in Ungarn oder Polen mehrfach erfolgreich gebeten wurde, an verschiedenen Ecken und Enden das Rechtsstaats-Gebäude per Volksabstimmung einzureißen. Die im Grunde unkörperlichen, ubiquitären Stimmen des neuen Regimes versuchen, eine Mauer

62 Wajsbrot, Cécile: Destruction, S. 163: „Les corbeaux envahissent la ville. Ils sont arrivés petit á petit, à mesure des années. Imperceptiblement.“

63 Ebda., S. 177. 
zwischen Vergangenheit und Gegenwart zu errichten, um die Zukunft zu kontrollieren. Cécile Wajsbrots dystopische Analyse demonstriert, wie die Vergangenheit Teil der Gegenwart ist, wie Fiktion sich mit Wirklichkeit verbunden hat und wie alle Rechtstaatlichkeit Stück für Stück aufgelöst wird.

Zahlreiche Grenzen werden auf dem Weg in die Diktatur sowie im Schlagschatten autoritärer Kontrollmechanismen überschritten. Im Fokus der um sich greifenden Zerstörung, die durch wohlorganisierte Massendemonstrationen untermauert wird, steht immer wieder die Sprache. Auf sie versucht man einzuwirken, um sie herum errichtet man Grenzen. Wozu noch Fremdsprachen lernen, so fragen die allgegenwärtigen Stimmen, wenn doch die eigene Sprache das wichtigste ist? Die Gegenwart hat uns gelehrt, mit der in einer Fiktion entfalteten Dummheit vorsichtig umzugehen, könnte sie doch in der Wirklichkeit noch deutlich größer sein.

Doch es geht auch um den Zustand der eigenen Sprache. Denn bezüglich der Muttersprache ist keine komplexes und vieldeutiges Idiom erwünscht, sondern eines, wie es für Ziele und Zwecke der Propaganda nützlich ist: Es geht um Sprache als Propagandavehikel, um die Beendigung jeglicher Polysemie durch Vereindeutigung und Sinnreduktion: „un véhicule utilitaire transportant leur propagande, un ensemble de mots á sens unique. “64 Hypotaktische Strukturen werden ausgemerzt, parataktische Strukturen auf die immer selbe Satzgliederung reduziert, komplexe Sinnbildungsprozesse wo möglich unterbrochen, andere Sprachen weitestgehend ausgeblendet, ältere Texte beseitigt: Es erfolgt eine linguistische Homogenisierung auf unterstem Niveau. Jede Möglichkeit, Komplexität zu denken, soll schon im Kern unterbunden werden. Da ist das Medium der Literatur, um welches es in Destruction geht, von einer Brisanz, gegen die sich jegliche Diktatur, jegliches autoritäre Regime zu verschwören sucht.

Neben der Kontrolle von Vergangenheit und Zukunft sowie von Sprache und damit Denken werden Gemeinschaftssinn und Disziplin, Loyalität und Treue gefördert und zugleich im Kampf gegen jegliche Dissidenz allen möglichen Formen autoritärer Überwachung einer Zensur, einer Zerstörung unterworfen, die ubiquitär zu sein scheint. Zensur ist überall: in den Medien, in den Köpfen. Überall wird „Platz geschaffen“, wird „ausgekehrt“ und „,sauber gemacht“: Überall werden Säuberungen durchgeführt und alles, was auf andere Möglichkeiten des Denkens und Handelns verweisen würde, ausgelöscht und zerstört. Denn das Schlüsselwort all dieser Entwicklungen ist Destruction, Zerstörung, die keine Spiel-Räume für Kreativität mehr lässt.

64 Ebda., S. 211. 
Das erste, was unwiederbringlich zerstört wird, ist - wie wir sahen - der unerschütterliche Glaube einer ganzen Generation, dass nach der Barbarei des Ersten und des Zweiten Weltkriegs, nach den unvorstellbaren und monströsen Taten der Shoah, nach den Diktaturen der Nationalsozialisten, aber auch vieler anderer faschistischer Bewegungen in Europa ein weiteres Abgleiten in eine autoritäre Herrschaft niemals mehr möglich sein würde. Nun, dieser Glaube ist geschwunden, ja mehr noch: Er ist zerstört. Nach einer am 13. Februar 2018 veröffentlichten Umfrage der Universität Bielefeld halten es knapp fünfzig Prozent der Deutschen wieder für möglich, dass sich ein Holocaust - also die Vernichtung der europäischen Juden - wiederholen könnte. ${ }^{65}$ Ist die Möglichkeit einer Insel nicht schon der wichtigste Schritt hin zur Existenz dieser Insel? Wie konnte es sein, dass das sicher Geglaubte sich binnen kürzester Frist aufgelöst hat?

In jedem Fall bleibt von den Glaubenssätzen der Generation der Ich-Erzählerin nichts mehr übrig, lehrt doch die lange nicht zu Bewusstsein kommende oder unterdrückte Einsicht in Veränderungen, welche eine neue Diktatur heraufgeführt haben, dass die Diktaturen aus der eigenen Geschichte definitiv nicht verschwunden sind. Dabei handelt es sich ganz offenkundig keineswegs um eine von außen kommende oder von fremden Mächten aufoktroyierte Gewaltherrschaft. Es ist vielmehr eine solche, die von innen kommt, die von vielen jener Menschen getragen wird, welche gestern noch die Sonnenfinsternis gemeinsam bejubelt haben.

Die Diktatur, deren Schergen meist zu dritt und bewaffnet ohne Vorankündigung in Privatwohnungen eindringen und nach Belieben Dokumente und Erinnerungen zerstören, die in Zivil in öffentlichen Transportmitteln patrouillieren und jedes Gespräch der zum Schweigen verurteilten Staatsbürger unterbinden, die alle Kulturveranstaltungen gegen nur anfänglichen Widerstand gleichgeschaltet haben und fortan effizient kontrollieren, ist zweifellos eine Gewaltherrschaft, die aus dem Volke kommt. Sie ist - parallel zur erwähnten Toynbee'schen These - aus der eigenen Gesellschaft entstanden und speist sich aus der wachsenden Absage an die vorherige, vermeintlich so stabile Gesellschaftsordnung. Es handelt sich, wie alle Stimmen im Roman zum Ausdruck bringen, folglich um eine selbstgewählte Diktatur, die sich offenkundig nach der Sonnenfinsternis der Jahrtausendwende ausgebreitet und alle Hoffnung darauf zerstört hat, dass sich die schrecklichen Erfahrungen des 20. Jahrhunderts niemals mehr wiederholen

65 Vgl. http://www.stiftung-evz.de/fileadmin/user_upload/EVZ_Uploads/Pressemitteilungen/ MEMO_PKfinal_13.2.pdf (S. 14). Stimme eher $\mathrm{zu}=26 \%$; stimme stark $\mathrm{zu}=22 \%$. Dr. Jonas Rees, Prof. Dr. Andreas Zick: Trügerische Erinnerungen. Wie sich Deutschland an die Zeit des Nationalsozialismus erinnert. Uni Bielefeld 2018. 
würden. Nun ist die Türe weit offen für einen neuen Zyklus von Gewalt, Krieg und rasender Zerstörung.

Wie ist all dies zu erklären? Und wie könnte eine derartige Entwicklung noch gestoppt werden? Welche Überzeugungen können noch an die Stelle der zerstörten Hoffnungen treten? Müssen es nun nicht Vorstellungen von zyklisch wiederkehrenden Heimsuchungen sein, von Entwicklungen, die - vergleichbar mit den im Text des Öfteren auftauchenden Naturkatastrophen, ${ }^{66}$ die freilich menschengemacht sind - die Menschen unausweichlich, wenn auch nicht unvermittelt treffen? Nichts war überraschend gekommen, nichts hatte sich wie eine plötzliche Plage über die Menschen hergemacht: Alles war vielmehr schon längst präsent gewesen. Es handelt sich um eine Präsenz der Vergangenheit im Präsens der eigenen Wahrnehmung:

Es war nicht von einem Tag auf den anderen gekommen, alles war schon da und wir hatten es nicht gesehen. Oder eher noch, wir hatten es gesehen, aber ohne daran glauben zu wollen. Und die Leere der Straßen an jenem Abend war das ureigene Bild unseres Desertierens in der letzten Zeit. Diese absoluten Regime, die weit entfernten Ländern bestimmt zu sein schienen, wohin wir niemals gehen würden oder die definitiv unserer Vergangenheit angehörten, werden wir sie nun unsererseits kennenlernen? Gab es in der Geschichte so etwas wie eine bestimmte Masse an Diktatur, welche immer dieselbe wäre und verteilt würde auf die Epochen und die Orte, einmal auf diesen Kontinent oder auf jene Hauptstadt? Aus war es mit der Kontemplation des Unglücks der anderen, seufzend zwar, aber gemischt mit ein wenig Erleichterung - es ist weit weg, es sind nicht wir -, bevor man zu etwas anderem überging und vergaß. Fortan wären es die anderen, die uns einen Moment lang beklagen würden, und wir, die wir nur ein wenig Mitleid abbekämen, ohne dass uns wirklich geholfen würde. ${ }^{67}$

Die Katastrophe, die immer schon da war, unter uns, und ohne dass wir ihr größere Aufmerksamkeit gewidmet hätten: Sind wir nur ganz einfach sorglos gewesen und gleichgültig, was das Los der anderen angeht? Die Wiederkehr der Diktatur, von der wir uns doch im Raum oder in der Zeit sicher getrennt glaubten, lässt Bilder einer regelmäßigen Wiederkehr autoritärer Regime entstehen, die sich gleichmäßig über die Geschichte und Kontinente verteilen. Gibt es eine Gesetzmäßigkeit in der Geschichte, der wir ganz einfach unterliegen?

66 Zur Semantik von Naturkatastrophen vgl. Ette, Ottmar: Carnival and other Catastrophes. New Orleans: A Global Archipelago. In: Ette, Ottmar / Müller, Gesine (Hg.): New Orleans and the Global South. Caribbean, Creolization, Carnival. Hildesheim - Zürich - New York: Georg Olms Verlag 2017, S. 15-67.

67 Wajsbrot, Cécile: Destruction, S. 74. 
Gewiss: Die Diktatur, die nun in Frankreich ausgebrochen ist, besitzt keinen eigentlichen Diktator, keinen „chef unique“, ist eine Diktatur ohne Diktator. ${ }^{68}$ Aber ihre radikale Umgestaltung der vorherigen Gesellschaft, ihre fundamentale Zerstörungswut, die sich gegen alles Vergangen-Präsente wendet, macht deutlich, dass man längst unter den Bedingungen einer allgegenwärtigen Diktatur lebt, die vor allem und an erster Stelle die von ihr gesetzten Grenzen zu sichern und zu überwachen sucht. Ob es nun einen klassischen Diktator gibt oder nicht ...

Die Literatur, die in einer solchen Zeit entsteht, gerät nicht zu einem simplen „Handbuch des Überlebens im diktatorischen Milieu“;;99 aber Züge eines derartigen Handbuchs des Überlebenswissens in diktatorischen Zeiten sind in Cécile Wajsbrots Destruction durchaus wahrnehmbar. Denn es geht in diesem Roman schlicht um die Aufgabe der Literatur, um ihre Rolle und Funktion gerade auch als prospektive Warnerin vor Entwicklungen, die noch unmerklich klein, aber bereits gegenwärtig sind: die unter uns sind, aber von den meisten nicht bemerkt werden wollen. Es geht um die Frage, welche Rolle der Literatur, den Literaturen der Welt in medial veränderten Gesellschaften zukommt, in welchen andere künstlerische Ausdrucksformen nicht über die prospektive, voraussehende Kraft des literarischen Wortes verfügen. Nicht umsonst hatte Cécile Wajsbrot weiteren künstlerischen Medien wie der Videokunst und Photographie, der Malerei oder der Bildhauerei die einzelnen Romane ihres Zyklus gewidmet.

Denn es geht in Destruction, seinem Schlussstein, um eine möglichst präzise Beobachtung der Veränderungen auf den Ebenen von Sprache und Verhalten, von politischem Diskurs und Schreibweise, von Symboliken und Ritualen, die sprachlich vermittelt sind. Das literarische Schreiben vermerkt seismographisch genau die kleinsten Veränderungen, die - um es mit einem noch vor Beginn des Zweiten Weltkrieges von Nathalie Sarraute geprägten und in unserer Vorlesung wiederholt behandelten Begriff zu sagen - kaum wahrnehmbaren „Tropismen“, ${ }^{70}$ die sich in alle Ausdrucks- und Handlungsebenen der Menschen einschleichen. Oft sind es feine und fast unmerkliche Bewegungen, welche Individuen wie Gemeinschaften über Grenzen bewegen: auch über die Tausenden von Grenzen zwischen Demokratie und Diktatur.

Weit mehr als ein Handbuch des Überlebenswissens in Zeiten beginnender Diktatur, bildet die Literatur dagegen ein künstlerisch hochdifferenziertes Frühwarnsystem, das die Zeichen der Zeit, die Vergangenheit in der Gegenwart, aber auch Vergangenheit und Gegenwart in der Zukunft befragt und analysiert. Es gibt

68 Ebda., S. 153.

69 Ebda., S. 145: „manuel de survie en milieu dictatorial.“

70 Vgl. Sarraute, Nathalie: Tropismes. Paris: Denoël 1939. 
kein anderes diskursives künstlerisches Medium, das wie die Literatur quer zu den Jahrtausenden und Jahrhunderten, quer $\mathrm{zu}$ den verschiedenartigsten Kulturen, quer zu den unterschiedlichsten Gesellschaftsschichten diese seismographische Aufgabe so erfüllen könnte. Daher ist sie für ein Überleben der Menschheit so wichtig, so überlebenswichtig. Dies wird in der im Roman bearbeiteten Diegese, mithin in unserem Raum, in unserer Zeit, nach Ende der vierten Phase beschleunigter Globalisierung und nach Ende der Postmoderne so deutlich wie nur selten zuvor.

Denn die Literaturen der Welt wenden sich seit ihren vielkulturellen Anfängen im Gilgamesch-Epos, seit ihren verdichteten lyrischen Formen im chinesischen Shi-Jing, nicht allein der Vergangenheit und ihrer Erinnerung zu, sondern entwerfen weit über die Memoria-Funktion hinaus mögliche Zukünfte von Gesellschaften, deren Veränderungen sie möglichst präzise registrieren und die sie in ästhetisch verdichteter Form sinnlich erfahrbar machen. Die Literaturen der Welt bieten uns ein LebensWissen und ÜberlebensWissen, das uns als ein Erlebenswissen nacherlebbar offeriert wird und uns in die Lage versetzen kann und soll, ein ZusammenLebensWissen auszuprägen und experimentell zu erproben.

Die Literaturen der Welt sind darum gerade heute für uns überlebenswichtig, weil sie uns sinnlich ins Bewusstsein rufen, wie prekär zu jedem Zeitpunkt die Gewissheiten sind, denen wir uns für die Dauer bestimmter Zeiträume anzuvertrauen pflegen. Sie zeigen uns nicht nur retrospektiv, sondern vor allem prospektiv auf, in welchem Maße es aufmerksam zu sein gilt, wenn wir uns im Grenzbereich und mehr noch im Spannungsfeld von Demokratie und Diktatur bewegen - so, wie wir dies in unserer Epoche auf gefährliche Weise auch in Europa tun.

Denn es wäre grundverkehrt, auf die eine Grenze zwischen Diktatur und Demokratie, zwischen Demokratie und Diktatur zu setzen. Keine Grenze, schon gar keine Mauer, nichts schützt uns vor dem unmerklich beginnenden Hereinbrechen einer Vergangenheit, die nicht mehr ist und doch nicht aufhören kann zu sein - wie es das „Paradigma Jauss“71 in unserer Zeit mit aller wünschenswerten Deutlichkeit bis heute zeigt. Die Vergangenheit ist gegenwärtig: auch in unserem Fach, der Romanistik.

Die Vervielfachung der Grenzen zwischen Demokratie und Diktatur führt uns zugleich vor Augen, dass wir ständig vervielfachte Grenzziehungen in diesem Spannungsfeld überqueren können, ohne uns stets dieser Tatsache bewusst sein zu müssen. Der Fall Jauss zeigte nicht nur den Fall des Hans Robert Jauss, sondern anhand anhaltender Auseinandersetzungen auch die

71 Vgl. Ette, Ottmar: Der Fall Jauss. Wege des Verstehens in eine Zukunft der Philologie. Berlin: Kulturverlag Kadmos 2016. 
paradigmatische Bedeutung dieses Falles, an dem sich höchst unterschiedliche Wertesysteme und Gesellschaftsentwürfe dokumentieren. Zugleich verweist er auf die Geschwindigkeit und Leichtigkeit, mit der sich Menschen zwischen der schlimmsten Barbarei und einer Nachkriegsdemokratie bewegen können, in der sie die Präsenz der Vergangenheit gegenwärtig halten. Denn das Schweigen und Verschweigen der Vergangenheit hält diese Vergangenheit in der Gegenwart präsent und bereitet diese Präsenz vor auf das Kommende, das Künftige, auf unsere Zukunft, die bedroht ist.

Der argentinische Schriftsteller Julio Cortázar hat in seiner Erzählung Casa Tomada vor dem Hintergrund der Militärdiktaturen seines Landes und seines Kontinents die vielen kleinen Grenzverletzungen und das fatale Überlassen von Räumen gezeigt, die letztlich dazu führen, dass eine Familie in ihrem Haus Raum um Raum aufgibt, bevor sie von ihrem Wohnsitz gänzlich vertrieben ist. In einer graphischen Umsetzung dieser Erzählung ${ }^{72}$ wird am Ende vorgeführt, wie die Worte des Erzählers zu Vertriebenen an ihrem eigenen Ort geworden sind, ohne doch $\mathrm{zu}$ wissen, wann alles begonnen hat. Ist erst einmal die Vertreibung aus dem Haus vollzogen, verwandeln sich die vielen Grenzen in eine stabile Grenzziehung des Ausgeschlossen-Seins. Dann erst schließen sich die Grenzen und lassen zur Katastrophe werden, was mit einem fast unmerklichen Eindringen begonnen hat. In einer Zeit, in der dieses Eindringen bereits massiver geworden ist und die alte Barbarei neuen Zulauf erhält, wächst die Aufgabe von Literatur und Literaturwissenschaft, Frühwarnsysteme im Angesicht kommender Bedrohung zu installieren und ein Lebenswissen zu entfalten, das seit allen Anfängen der Literatur, seit den Erzählungen von 1001 Nacht, immer auch ein ZusammenLebensWissen ist.

Denn die prospektive Macht der Literaturen der Welt zeigt uns immer wieder in aller Deutlichkeit auf, wie nahe uns das vermeintlich in Raum und Zeit so Distante ist, wie wachsam es zu sein gilt, um das Verdrängte, das nur scheinbar verschwunden ist, an der Wiederkehr durch kritische Auseinandersetzung zu hindern. Denn was nicht mehr ist, war doch einmal vergangene Zukunft und trägt diese Potentialität noch immer gerade in dem, was wohl niemals aufhören kann zu sein. Die Literaturen der Welt zeigen eindrucksvoll, dass das Vergangene niemals vergangen, sondern stets gegenwärtig ist und nicht aufhören kann zu sein. Literatur selbst ist durch ihr intertextuelles Verwoben-Sein stets an Vergangenheiten gebunden, welche in Gegenwart und Zukunft projiziert werden und die Vernetzung aller Zeitebenen sinnlich und lebensnah vor Augen führen.

72 Cortázar, Julio: Casa Tomada. Diseño gráfico por Juan Fresán. Buenos Aires: Ediciones Minotauro 1969. 
Im lebendigen, interaktiven Archiv und Archipel der Literaturen der Welt verfügen wir quer zu den Räumen, den Zeiten und auch den Regierungsformen über ein Wissen, das mit unserem ,realen' Leben nicht gleichzusetzen und doch von diesem nicht zu trennen ist. Dieses Wissen für Gegenwart und Zukunft des Menschen zu erschließen, ist die Aufgabe der Literaturwissenschaft, die Aufgabe der Philologie.

Anders als die Literaturwissenschaft nimmt die Literatur längst ihre genuinen Aufgaben wahr. Spätestens seit dem Wechsel des Jahrtausends - wenn auch nicht unbedingt seit der von Cécile Wajsbrot ins Feld geführten Sonnenfinsternis von 1999 - zeigen uns die Literaturen der Welt jene Bedrohungsszenarien auf, gegen die anzugehen noch Zeit ist, eine Zeit, die freilich schwindet. Mit dem Ende der vierten Phase beschleunigter Globalisierung um die Mitte des zweiten Jahrzehnts unseres Jahrhunderts haben sich diese Stimmen in den Literaturen auf eine Weise verstärkt, welche endgültig die Zeiten im Zeichen der Postmoderne historisch werden ließ. Vor uns liegen die Archipele der Zukunft, die freilich jederzeit zu in sich abgeschlossenen Insel-Welten mutieren können. Doch ist die Offenheit dieser archipelischen und transarchipelischen (und damit relationalen) Inselwelten ein Versprechen auf eine viellogische Zukunft, in der uns die Literaturen der Welt mit ihrer polylogischen Mobilität, quer durch Räume und Zeiten, Sprachen und Kulturen, als Erprobungsräume des Künftigen begleiten werden. An der Schwelle zu den historischen Avantgarden warnte Alfred Jarrys Ubu Roi vor den Kriegen und Massakern des künftigen Jahrhunderts. Auch in unserem Jahrhundert, nach einer weiteren Phase beschleunigter Globalisierung, nach den Zeiten der Postmoderne und inmitten der Zeichen künftiger Konvivenz und künftiger Katastrophen ist Ubus Stimme, sind die Stimmen dieser Geschichte der Literaturen der Welt keineswegs verklungen. Vielperspektivisch weisen sie auf die Orte und mehr noch auf die Bewegungen der Menschen im Universum. 